Mon. Not. R. Astron. Soc. 000,118(2017) Printed 25 February $2021 \quad$ (MN LATEX style file v2.2)

\title{
A comparative analysis of Galactic extinction at low Galactic latitudes
}

\author{
A.C. Schröder, ${ }^{1 \star}$, W. van Driel ${ }^{2,3}$, R.C. Kraan-Korteweg ${ }^{4}$ \\ ${ }^{1}$ South African Astronomical Observatory, PO Box 9, Observatory 7935, Cape Town, South Africa \\ ${ }^{2}$ GEPI, Observatoire de Paris, PSL Research University, CNRS, 5 place Jules Janssen, 92190 Meudon, France \\ ${ }^{3}$ Station de Radioastronomie de Nançay, Observatoire de Paris, CNRS/INSU USR 704, Université d'Orléans OSUC, \\ route de Souesmes, 18330 Nançay, France \\ ${ }^{4}$ Department of Astronomy, University of Cape Town, Private Bag X3, \\ Rondebosch 7701, South Africa
}

Accepted....... ;

\begin{abstract}
We use near-infrared $(J-K)$-colours of bright 2MASS galaxies, measured within a $7^{\prime \prime}$-radius aperture, to calibrate the Schlegel et al. (1998) DIRBE/IRAS Galactic extinction map at low Galactic latitudes $\left(|b|<10^{\circ}\right)$. Using 3460 galaxies covering a large range in extinction (up to $A_{\mathrm{K}}=1.15$ or $E(B-V) \simeq 3.19$ ), we derive a correction factor $f=0.83 \pm 0.01$ by fitting a linear regression to the colour-extinction relation, confirming that the Schlegel et al. maps overestimate the extinction. We argue that the use of only a small range in extinction (e.g., $A_{\mathrm{K}}<0.4$ ) increases the uncertainty in the correction factor and may overestimate it. Our data confirms the Fitzpatrick (1999) extinction law for the $J$ - and $K$-band. We also tested four all-sky extinction maps based on Planck satellite data. All maps require a correction factor as well. In three cases the application of the respective extinction correction to the galaxy colours results in a reduced scatter in the colour-extinction relation, indicating a more reliable extinction correction. Finally, the large galaxy sample allows an analysis of the calibration of the extinction maps as a function of Galactic longitude and latitude. For all but one extinction map we find a marked offset between the Galactic Centre and Anticentre region, but not with the dipole of the Cosmic Microwave Background. Based on our analysis, we recommend the use of the GNILC extinction map by Planck Collaboration et al. (2016b) with a correction factor $f=0.86 \pm 0.01$.
\end{abstract}

Key words: ISM: dust, extinction - galaxies: photometry - infrared: galaxies

\section{INTRODUCTION}

Accurate photometric measurements of galaxies, like brightness or colours, and their derived quantities, for instance stellar masses and star formation rates, require a correction for Galactic foreground extinction. While such a correction is usually small and often negligible at high Galactic latitudes, it becomes more and more important at lower latitudes and starts affecting completeness estimates and other statistical evaluations as well.

The first, frequently used, extinction map was based on H I column density measurements and galaxy counts, assuming a correlation between gas and dust in the interstellar medium (Burstein \& Heiles 1978, Burstein \& Heiles 1982). More recently, Lenz et al. (2017) have published a new map

* E-mail:acs@saao.ac.za based on H I column densities, albeit covering only the lowcolumn density, high-latitude sky.

However, since the gas-to-dust ratio is not constant everywhere and can vary by up to a factor of two locally (e.g., Burstein et al. 1987), more accurate extinction maps, based on far-infrared (FIR) dust emission, were introduced by Schlegel et al. (1998, hereafter SFD), using high-resolution all-sky maps from the DIRBE and IRAS missions. The advantage of these maps is that they cover all Galactic latitudes though they could not be calibrated in the Galactic plane $\left(|b|<5^{\circ}\right)$ due to FIR confusion problems and lack of known galaxies.

There have been various attempts to calibrate the SFD maps in general (e.g., Dutra et al. 2003 with a correction factor $f=0.75$, Choloniewski \& Valentiin 2003 with $f=0.71$ ) and at low Galactic latitudes (the so-called Zone of Avoidance, hereafter ZoA) in particular (e.g., Nagavama et al. 
2004 with $f=0.67$, Schröder et al. 2007 with $f=0.87$ ). In 2011, Schlafly \& Finkbeiner (hereafter SF11) have done a more comprehensive investigation and derived a correction factor $f=0.86$ for the all-sky SFD maps that has been widely accepted.

Recently, the Planck mission with a more sensitive survey of the infrared sky has produced several whole sky extinction maps, some based on the first data release (PR1; Planck Collaboration et al. 2014, Meisner \& Finkbeiner 2015), others on the full mission data (PR2; Planck Collaboration et al. 2016, Planck Collaboration et al. 2016). Although there have been various attempts in comparing the available extinction maps (e.g., Chiang \& Ménard 2019), most comparisons were done at high latitudes or low extinction levels.

We have therefore decided to use a comprehensive and magnitude-limited sample of near-infrared (NIR) bright galaxies from the 2MASX catalogue at low latitudes $(|b|<$ $\left.10^{\circ}\right)$ and high extinction levels elsewhere $\left(E(B-V)>0 .^{\mathrm{m}} 95\right.$; Schröder et al. 2019, hereafter Paper I) to investigate the existing extinction maps using the galaxy colours. Our main aim was to derive a correction factor that is also valid at high extinction levels and to search for possible variations across the Galactic plane region. We have thus determined correction factors for both the SFD map and the four Planck mission maps.

This paper is structured as follows. We introduce the galaxy input sample used for our analysis in Section 2 Section 3 presents the colour-extinction relation and investigates ways to construct the cleanest possible sample and best parameter settings. In Section 4 we investigate the four extinction maps based on Planck data, and in Section 5 we study the variation of the calibration factor across the ZoA. We summarise our findings and give conclusions in Section 6 . Throughout the paper, $K$ refers to the 2 MASS $K_{\mathrm{s}}$-band, and the superscript 'o' indicates a correction for extinction.

\section{THE SAMPLE}

Paper I gives a detailed description of the selection criteria for our galaxy catalogue, but for easy reference we recapitulate the most important criteria here.

We selected all 2MASX1 objects that satisfy the following criteria:

(i) $K^{\mathrm{o}} \leq 11^{\mathrm{m}} 25$ (i.e., corrected for SFD Galactic extinction);

(ii) $|b| \leq 10^{\circ}$ or $E(B-V)>0 .{ }^{\mathrm{m}} 95$.

To correct for Galactic extinction we used the SFD maps without any correction factor so as to be comparable to the 2MASS Redshift Survey (2MRS; Huchra et al. 2012) selection criteria. We visually inspected all objects using images from various passbands (optical to NIR) to distinguish between galaxies, blended stars, artefacts and Galactic nebulae. This resulted in 3675 plus 88 galaxies for the 2MZOA $\left(|b| \leq 10^{\circ}\right)$ and $2 \mathrm{MEBV}\left(E(B-V)>0 .^{\mathrm{m}} 95,|b|>10^{\circ}\right)$ catalogue, respectively.

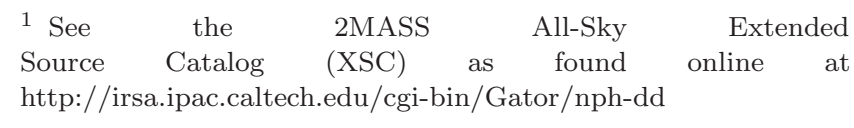

Table 1. NIR extinction coefficients relative to the $B$-band

\begin{tabular}{cccc}
\hline Passband & $\begin{array}{c}\lambda \\
(\mu \mathrm{m})\end{array}$ & $\begin{array}{c}\text { Fitzpatrick 1999 } \\
A_{\lambda} / A_{\mathrm{B}}\end{array}$ & $\begin{array}{c}\text { Cardelli et al. 1989 } \\
A_{\lambda} / A_{\mathrm{B}}\end{array}$ \\
\hline$J$ & 1.25 & 0.208 & 0.211 \\
$H$ & 1.65 & 0.128 & 0.136 \\
$K_{\mathrm{S}}$ & 2.15 & 0.087 & 0.085 \\
\hline
\end{tabular}

For the our investigation, we excluded four galaxies that have an unknown galaxy classification (class 5 in Paper I) and 166 galaxies where the 2MASX fit is centred on a superimposed star instead of the galaxy's bulge - we assume all the photometry of these objects to be unreliable. That results in an input sample of 3593 galaxies. This input sample has been carefully investigated and the resulting final sample, as detailed in the following section, comprises 3460 galaxies.

\section{GALACTIC EXTINCTION CORRECTION}

We use the method of calculating the extinction correction factor using NIR colours as outlined in Schröder et al. (2007). We first apply a correction for Galactic extinction using the SFD values (given as $E(B-V)$ ), $R_{V}=3.1$ (or $\left.R_{B}=4.14\right)$ and the Fitzpatrick 1999 extinction law (hereafter F99, see Table 1), resulting, for example, in a measured $A_{\mathrm{K}}=4.14 \cdot 0.087 \cdot E(B-V)$. If we then assume that the true extinction $\widetilde{A}_{K}$ is a constant $f$ times the measured value from the extinction map used, e.g., $\widetilde{A}_{K}=f A_{K}$, the slope $a$ of the relation between extinction-corrected NIR colours and extinction can be used to calculate $f$ as

$f=1+\frac{a}{E / A_{K}}$.

where $E$ is the colour excess of the respective colour. If the SFD maps were correct, the factor would be 1 .

As explained in detail below, we decided to use $(J-K)^{\circ}$ colours from the $7^{\prime \prime}$-aperture for this investigation. In addition to the selection criteria for the input sample mentioned in Sec. 2 we 'cleaned' the sample by deselecting on the 2MASX photometry Flag 4 and by excluding galaxies with excessive photometric uncertainties $\left(J\right.$-band error $\geq 0 .{ }^{\mathrm{m}} 13$, $K$-band error $\geq 0$. 12 ), hereafter called photometric selection criteria. We also excluded very high extinctions, setting the limit at $A_{\mathrm{K}}=1 . \mathrm{m} 15$.

Figure 1 shows the final 'cleaned' sample (green dots) and the linear regression fit; black dots show the input sample (without the photometric selection criteria applied and with no cut-off in extinction). The cleaned sample comprises 3460 galaxies and the linear regression fit gives

$\left(J-K_{\mathrm{s}}\right)^{\mathrm{o}}=(-0.241 \pm 0.012) \cdot A_{\mathrm{K}}+(1.036 \pm 0.004)$,

with a standard deviation or scatter of $0 . \mathrm{m}^{\mathrm{m}} 132$. This results in a correction factor of $f=0.83 \pm 0.01$.

In the following, we will discuss the individual selection criteria in detail.

2 Note that most red outliers are caused by a combination of wrong extinction values and photometric problems, cf. Figs. 24 
A comparative analysis of Galactic extinction

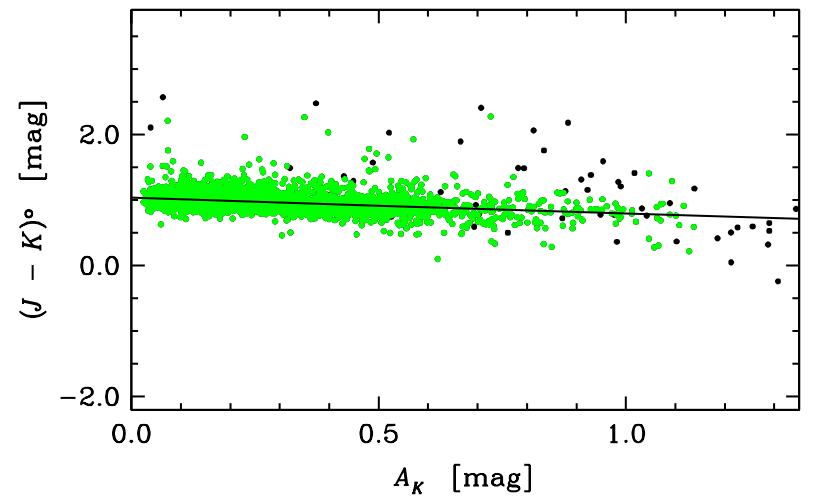

Figure 1. $7^{\prime \prime}$-aperture $(J-K)^{\circ}$-colour versus $K$-band extinction $A_{\mathrm{K}}$ (green dots) and linear fit for the cleaned sample. The black dots show the input sample before the selection criteria for the final sample were applied.

\subsection{Colour selection}

With three passbands one can construct three colours. The $(J-K)$-colour spans the largest wavelength range and is therefore the most reliable, as we have confirmed in our tests. However, for internal consistency we have conducted our investigation on the selection criteria using all three colours (for example, it helps to understand whether an outlier is due to problems in one passband only), but to avoid repetition, we present our results using only the $(J-K)$-colour.

\subsection{Aperture selection}

The 2MASX catalogue offers a variety of photometric measurements, ranging from small to large apertures, circular and elliptical apertures, isophotal and total magnitudes, as well as peak and central surface brightnesses. Since we are interested in the most stable colours, as little affected by contaminating stars as possible, we prefer a smaller circular aperture and therefore investigated those with radii $5^{\prime \prime}$, $7^{\prime \prime}$ and $10^{\prime \prime}$, hereafter A5, A7 and A10. For completeness reasons, we also looked at the isophotal magnitudes measured within the $K$-band $20 \mathrm{mag} \operatorname{arcsec}^{-2}$ isophotal elliptical aperture (hereafter ISO) as well as the central surface brightness (within a radius of $5^{\prime \prime}$; hereafter S5) and the peak pixel brightness (hereafter PK). Note that the latter two do not have errors or flags associated with them.

While we want to use as clean a sample as possible, we also want to retain as many galaxies as possible for the subsequent investigation by region. The two requirements are contrary, that is, we need to balance the benefits of one against the detrimental effects of the other. To start with, we find that up to 172 galaxies have no measurement in at least one band per investigated aperture. This favours A7, where all galaxies have measurements at all bands. Furthermore, bands and apertures are differently affected by large uncertainties and flag settings (see also the following sections). We found that 2MASX Flag 4 affects more objects the larger the aperture, which favours A5 magnitudes. Regarding the magnitude error distributions, we find that the isophotal magnitudes have on average $2-3$ times larger uncertainties than the aperture magnitudes, which argues against using these magnitudes (apart from the obvious problem of in- creased contamination with superimposed stars). The error distributions for the three aperture magnitudes, on the other hand, are quite comparable.

Studying the individual linear fits, we find that the slopes depend little on the chosen magnitude and usually agree within the 1- $\sigma$ error. The offsets, however, are more strongly dependent on the aperture, implying a colour growth curve across galaxies, in which isophotal colours show the bluest colours (about 1 m $^{\mathrm{m}} 00$ for $(J-K)^{\circ}$ ) and PK colours are reddest (about $1^{\mathrm{m}} 09$ for $\left.(J-K)^{\circ}\right)$. The scatter (standard deviation of the sample) decreases with aperture size (from 0 . 173 for PK to $0{ }^{\mathrm{m}} 141$ for A5 to $0{ }^{\mathrm{m}} 129$ for ISO), but at the same time we have about 200 galaxies fewer for ISO than for A5 and A7 due to the increased problems with the photometry. Despite these variations, the $f$-value itself is fairly independent on the aperture used and varies only by about 0.01 .

Consequently, we decided to use A7, comprising the most galaxies and being a good compromise between contamination by stars and the amount of scatter. The arguments against isophotal colours are the smaller number of galaxies available and larger uncertainties on average, though their colours are truest to the galaxies. Surface brightness colours, taken from the same aperture as the A5 colours and having no associated errors, are quite similar to A5 but are not available for all the galaxies. The peak pixel brightness magnitudes are the worst with the largest scatter and reddest colours, being strongly subjected to statistical or noise fluctuations in a single pixel.

\subsection{MASX photometry errors}

Regarding photometry errors, we found that a cut at the tail end of the error distribution removes the worst outliers in the colour-extinction relation. Figure 2 shows as an example the $J$ - and $K$-band error distributions and the colour-extinction relation for $(J-K)$ using A7. If we cut off at a smaller limit (e.g., err $_{\text {lim }}^{K}=0.07$ and err $_{\text {lim }}^{J}=0.10$, magenta arrows), we lose too many 'good' objects together with only a few more outliers as compared to the more relaxed cut, as shown by the magenta crosses in the bottom panel. We thus decided to use the more relaxed cuts that clearly remove outliers without losing too many good data points, that is, $\operatorname{err}_{\lim }^{K}=$ $0 .{ }^{\mathrm{m}} 12, \operatorname{err}_{\mathrm{lim}}^{J}=0.13$ and $\operatorname{err}_{\text {lim }}^{H}=0 .{ }^{\mathrm{m}} 10$.

\subsection{MASX photometry flags}

The 2MASX confusion flags mostly refer to masked pixels or subtracted point sources within the relevant aperture. We have investigated the distribution of objects with a specific flag set in the colour-extinction relations and find that only Flag 4 ('aperture contained pixels within bright star mask') seems to indicate outliers without affecting too many good sources. We thus exclude all photometry where this flag was set. This affected about 15 to 180 objects depending on aperture and colour.

\subsection{MZOA catalogue information}

The 2MZOA and 2MEBV catalogues list various information regarding the quality of data. As mentioned above, we 

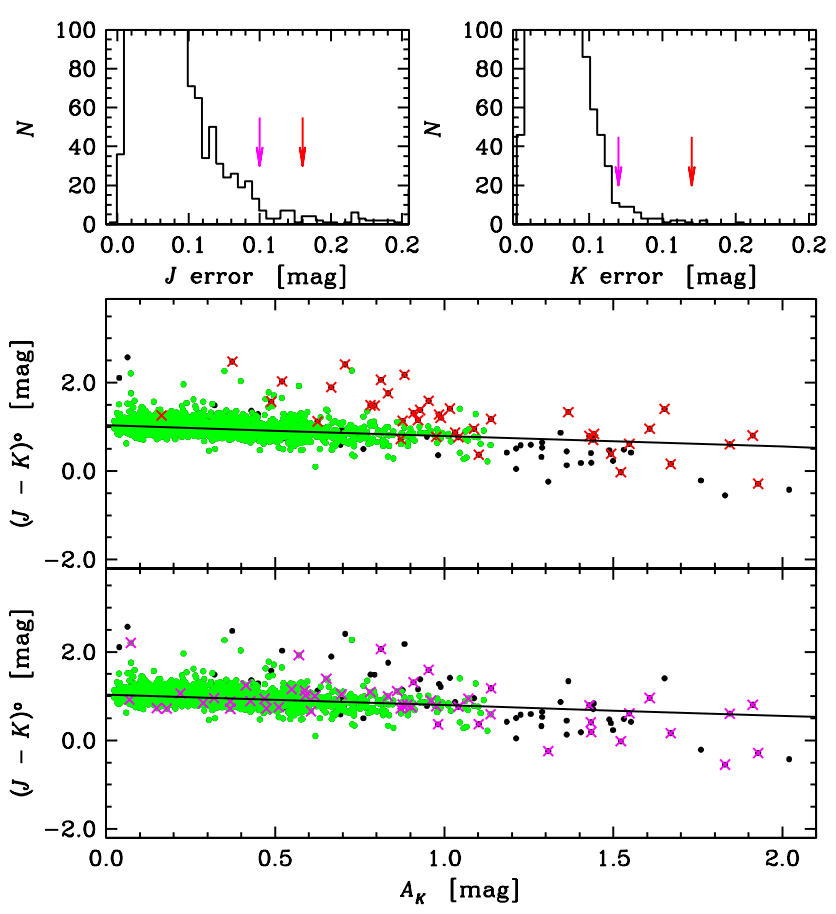

Figure 2. Testing for sample selection on photometry errors for A7. Top: truncated histograms of the error distribution for the $J$ band (left) and $K$-band (right); the arrows indicate the two cuts tested in the panels below. Middle: Colour-extinction relation for $(J-K)^{\mathrm{o}}$; black and green dots are the same as in Fig. 1 red crosses: objects with photometry errors above the higher cuts (see red arrows in the top panels). Bottom: Same as above but the magenta crosses indicate objects with photometry errors between the magenta and red arrows.

have excluded already the four objects which may or may not be galaxies, and all objects which are not centred properly on the galaxy's bulge. In addition, one of the flags indicates whether the extinction around a galaxy appears unreliable, a question that is of strong concern to us. However, this flag is only indicative for a strong variation in the overall area $\left(r \simeq 5^{\prime}\right)$ and not of the immediate environment of the galaxy and is by no means complete. While applying a cut on these flags removes a few outliers, too many good sources are affected as well $(N=101$ in case of A7), see Fig. 3. On the other hand, the linear regression parameters are little affected: excluding objects with this flag set changes the $f$ value by $\leq 0.003$, depending on aperture, and not at all for A7. We therefore decided not to use this flag and instead to rely on the conservative cut in $A_{\mathrm{K}}$.

\subsection{Extinction cut-off}

Figure 4 shows the $(J-K)$ colour distribution of the input sample (black dots) over the full extinction range. As in Fig. 11 the regression line is based on the cleaned sample (green dots). The colours of highly obscured galaxies become increasingly bluer, indicating a selection bias: in very highextinction regions, the extinction can vary spatially very rapidly and we are more likely to find galaxies in the lower extinction pockets. Due to the coarse spatial resolution of $6^{\prime}$ of the extinction maps, these are consequently corrected with a too-high, average extinction value and hence appear

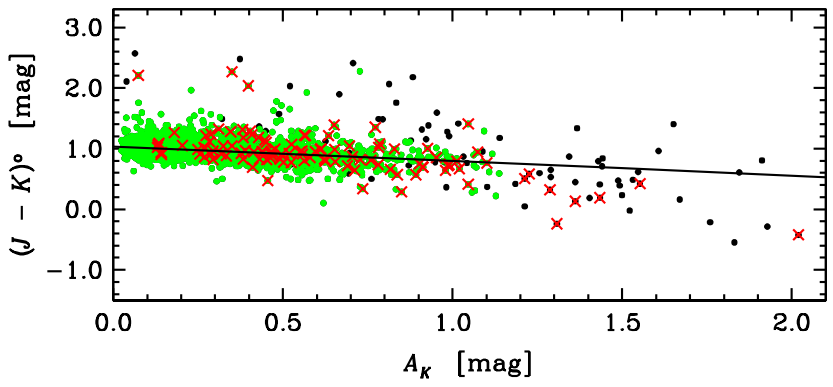

Figure 3. Testing selection on extinction flags for A7, Same as Fig. 1] with red crosses indicating objects with extinction flag ' $e$ ' set.

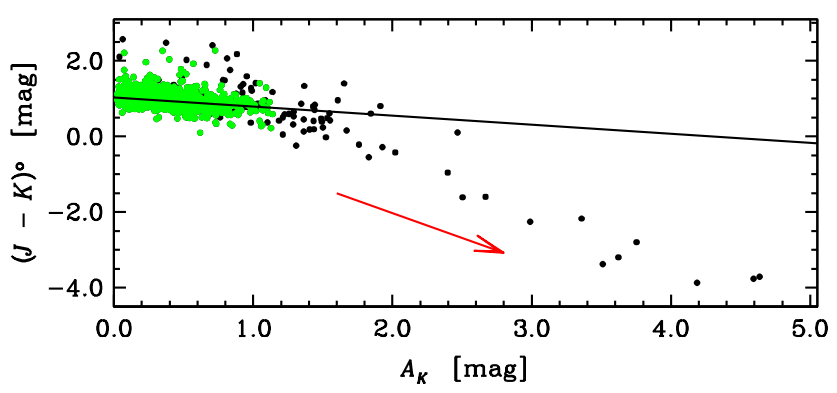

Figure 4. Same as Fig. 1 but showing the full extinction range. The reddening path is indicated with a red arrow.

too blue. The deviation increases with extinction, resulting in a non-linear relation. This effect has already been noted by, e.g., van Driel et al. (2009).

To determine the best cut-off in extinction to avoid this bias for our sample, we have used a series of upper limit values and determined each time the $f$-value, see Fig. 5 We find a plateau in the range $A_{\mathrm{K}}=0^{\mathrm{m}} \cdot 5-1{ }^{\mathrm{m}} \cdot 2$. The lower $f$-values at higher extinctions are clearly caused by the selection bias, while the higher $f$-values at the low-extinction end are likely due to a large extrapolation beyond the small range in extinction available for the fit and therefore being less certain, as implied by the larger error bars.

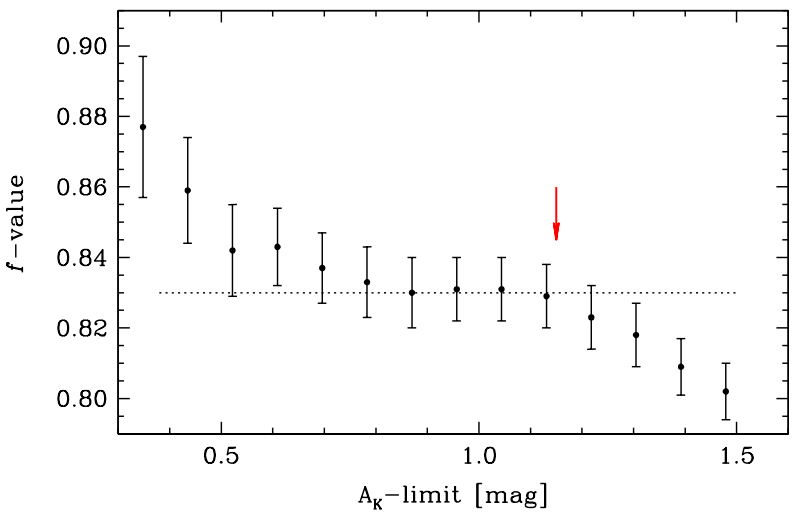

Figure 5. $f$-values derived for various upper limits in $A_{\mathrm{K}}$ for the linear regression fit. The dotted line shows the plateau at $f=0.83$ and the arrow the chosen cut-off in $A_{\mathrm{K}}$. 
A comparative analysis of Galactic extinction

Table 2. Comparison of $f$-values for different extinction laws for A7

\begin{tabular}{lccccc}
\hline Colour & F99 & C89 & Test & $\begin{array}{c}\text { F99 } \\
\text { 2-digit precision }\end{array}$ & $\begin{array}{c}\text { typical } \\
\text { error }\end{array}$ \\
\hline$(J-K)^{\circ}$ & 0.827 & 0.794 & 0.827 & 0.834 & 0.009 \\
$(J-H)^{\circ}$ & 0.817 & 0.871 & 0.838 & 0.817 & 0.006 \\
$(H-K)^{\circ}$ & 0.873 & 0.702 & 0.833 & 0.895 & 0.005 \\
\hline
\end{tabular}

\subsection{Other outliers}

Despite the 'cleaning' of the sample some outliers remain, likely due to insufficient star subtraction or other problems with an automated pipeline as was used for the 2MASX catalogue. Short of re-doing the photometry by hand, we have no simple and reliable selection criteria to get rid of these. Instead, we tested their effect on the linear regression by removing all data points outside a 4 - and a $3-\sigma$ envelope to the regression line. In case of A7, 22 and 54 data points, respectively, were thus removed. The effect is small, the $f$ value is $\sim 0.005$ smaller in case of a $4-\sigma$ cut, and 0.001 higher for the $3-\sigma$ cut. The other apertures give similar results. The deviations are smaller than the error on the $f$-value and we conclude that our linear regression is robust with respect to outliers.

\subsection{Extinction law}

As mentioned above, we have used the F99 extinction law to calculate the NIR extinction coefficients. To understand the impact of possible uncertainties in these coefficients on our results, we have tested the commonly used coefficients given by Cardelli et al. 1989, hereafter C89), see Table 1

Theoretically, the $f$-value should be independent of the colour used, and the variations we find between the fits for the three available colours are likely due to uncertainties in the colour measurements. These variations, however, are noticeably larger if we use the C89 extinction coefficients ( $\Delta f=0.17$ versus 0.06 for F99), see Table 2 hence we prefer the F99 coefficients. Nonetheless, while the $f$-values for the latter agree well for the colours $(J-K)$ and $(J-H)$, the $f$-value for $(H-K)$ still deviates. We can take this further and try to achieve better agreement between the colours: since mainly the $H$-band is affected, we varied its coefficient while leaving the others fixed. We find very good agreement within the error if we use a coefficient of 0.130 instead of 0.128 (named 'Test' in Table 2).

Note that the disagreements also increase when rounding the coefficients to the 2-digit precision used in Paper I. Considering the sensitivity of the $f$-value to the precision and exact value of the extinction coefficients, we recommend to use the higher precision values to avoid any possible systematics, even though they may be small.

To calculate the $K$-band extinction we use $R_{V}=3.1$ as recommeded by SFD. In particular in the Galactic plane, though, $R_{V}$ can vary: towards the Galactic bulge it seems to be lower $\left(R_{V}=2.5\right.$, Nataf et al. 2013), while the centres of molecular clouds can show values up to $4-6$. A change in $R_{V}$ results in a stretching of the colour-extinction relation, but only few data points would be affected (since we do not have many galaxies in the Galactic Bulge area).

\subsection{Correction for galaxy radius}

While smaller galaxies can be mostly or even fully contained in a fixed-radius aperture, for the largest galaxies a small aperture may contain only the bulge and therefore appear redder. Plotting colours versus major axis radii we find a slight dependence of up to $+0.0007 \pm 0.0001$ for $(J-K)$-colours, with the worst case for PK and being generally larger for the smaller apertures; ISO colours, on the other hand, show the reverse effect with a slope of $-0.0004 \pm 0.0001$. The effect starts disappearing when we remove all galaxies with radii smaller than around $15^{\prime \prime}$. Applying a correction for this dependence, however, has a negligible effect on the colour-extinction relation, mainly because the small galaxies are evenly distributed across the range of extinctions. It should be noted that there is also a dependence on morphological type on which we have little information. Ideally, we should use an aperture size that includes only light from the bulges (where available) and thus would vary from galaxy to galaxy. However, as already mentioned, since the effect is negligible and other uncertainties dominate the error budget, we have not applied a correction for this.

\subsection{Correction for stellar density}

In Paper I, we found that the colours show a slight dependence on stellar densities. We have repeated the investigation for all apertures and find that the effect on the determination of the $f$-value is negligible. While the dependence of colour on star density is $-0.078 \pm 0.010$ for the isophotal colours and smaller for the other apertures (except PK), e.g., $-0.037 \pm 0.011$ for $\mathrm{A} 7$, the change in the $f$-value is similar for all apertures and smaller than the 1- $\sigma$ error of 0.009 : for A7, $f$ increases from 0.828 to $f=0.835$ (for $N=3425$ since not all galaxies have a stellar density defined). Since the effect is small and to avoid introducing an additional uncertainty through the star density-colour relation, we have not applied such a correction.

\subsection{1 k-correction}

Since redshift changes a galaxy's colour (in $(J-K)$ they become redder), we have applied a k-correction to those galaxies that have redshift information and investigated the effect on the $f$-value measurement. We use the k-correction given by Chilingarian et al. (2010) who made their script available onlint $3^{3}$. The $\mathrm{k}$-correction depends on the colour of a galaxy, but instead of using the individual colours, we adopted generic colours: $(J-K)^{\circ}=1{ }^{\mathrm{m}} 0,(J-H)^{\circ}$ $=0.7,(H-K)^{\mathrm{o}}=0^{\mathrm{m}} 3$ (see Paper I). This avoids additional uncertainties due to errors in one of the passbands which slightly increase the scatter in the colour-extinction relation (from 0.122 to 0.128 ). The maximum difference in the two k-correction versions is \pm 0 . 02 for $(J-K)$, with a mean difference of $0.0003 \pm 0.0002$. The $\mathrm{k}$-corrections and adopted velocities used are given in a table described in the appendix.

We find that the k-correction has a negligible effect on 


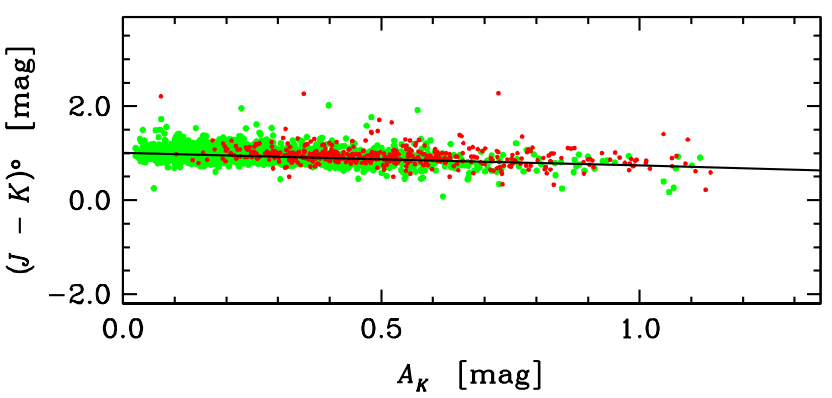

Figure 6. Same as Fig. 1 where green dots depict galaxies with redshift information and red dots galaxies without redshifts. The regression line was fitted to the green dots.
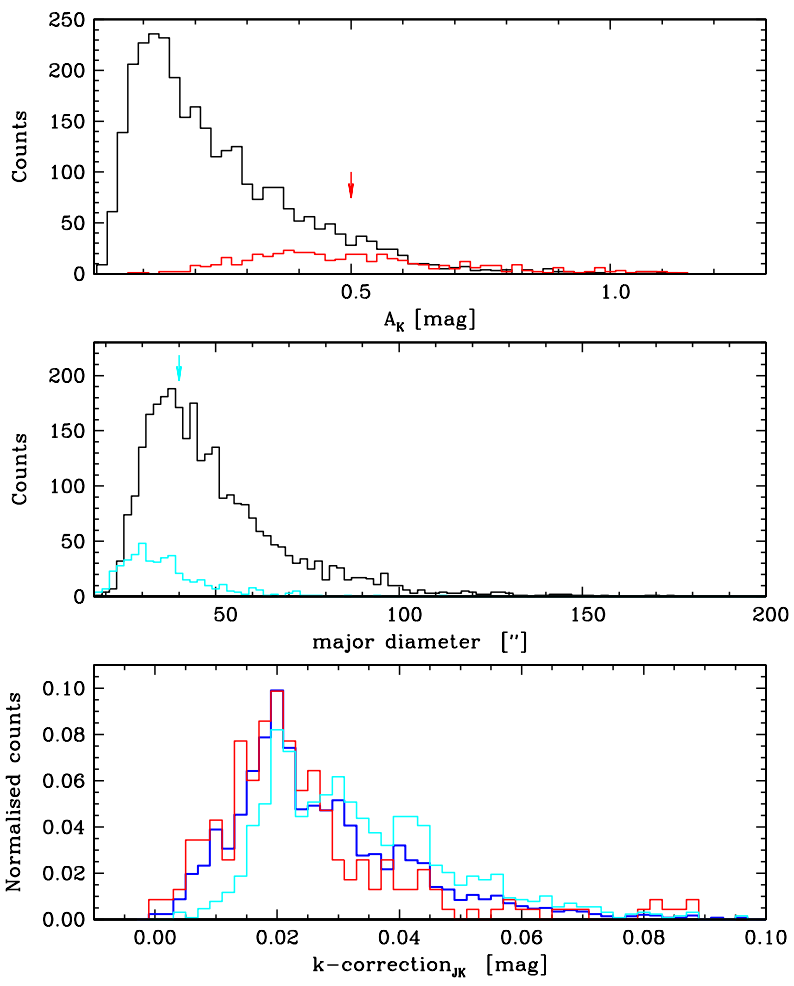

Figure 7. Distributions of galaxies with extinction $A_{\mathrm{K}}$ (top), major axis diameter $a$ (in arcseconds, middle) and k-correction in $(J-K)$ (bottom). The full sample is shown in black, galaxies with redshift information in dark blue, and galaxies with no redshifts in red (top) and cyan (middle), respectively. The bottom panel shows the samples after the application of the respective cuts indicated by the arrows and colours in the top and middle panel. For more information see text.

the determination of the $f$-value. However, not all galaxies have a redshift measurement (about 13\% are still missing, see the red dots in Fig. 6), and the reduced sample leads to a lower $f$-value of $0.801 \pm 0.010$. The same trend is found for all apertures. To understand better where the selection bias comes from and how it may affect the result if we had redshift measurements for all galaxies, we investigated further.

First, we looked at the distribution of galaxies with and without redshift measurements as a function of extinction and major axis diameter, both of which have a likely influence on whether a redshift was attempted to be measured.

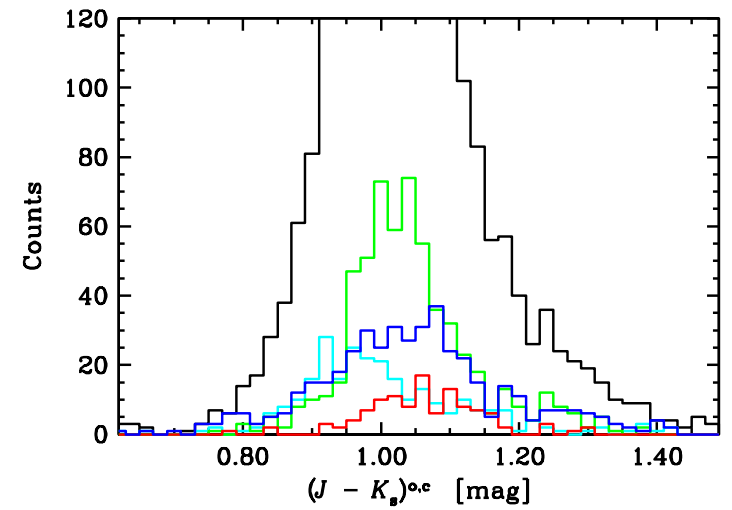

Figure 8. Colour distribution of galaxies with various velocity ranges: all galaxies (black), $c z<3000 \mathrm{~km} \mathrm{~s}^{-1}$ (cyan), $8000 \mathrm{~km} \mathrm{~s}^{-1}$ $<c z<11000 \mathrm{~km} \mathrm{~s}^{-1}$ (green), $15000 \mathrm{~km} \mathrm{~s}^{-1}<c z<25000 \mathrm{~km} \mathrm{~s}^{-1}$ (red), no velocity (dark blue).

The top two panels in Fig. 7 show the respective distributions for the full sample in black and for the sample without redshift information in colour. As expected, redshifts are predominantly lacking for galaxies at higher extinctions and having smaller observed diameters. The bottom panel, showing normalised counts for better comparison, presents the distribution of the k-correction values for all galaxies with a redshift (in dark blue) as well as for those after the application of an additional cut in extinction (red: $A_{\mathrm{K}}>0 .{ }^{\mathrm{m}} 5$ ) or in diameter (cyan: $a<40^{\prime \prime}$ ) applied, as indicated by the arrows in the upper panels. While the blue and red histograms compare reasonably well, the cyan histogram shows a clear shift (by about 0.005 ) with respect to the blue one. This means that galaxies at high extinctions do not show any preference in distance (as expected) and, consequently, in k-correction, but smaller galaxies tend to have a slightly higher k-correction because many are more distant, normalsized galaxies. Nonetheless, the smaller galaxies are evenly distributed over extinctions and therefore do not introduce an extinction-dependent bias in the colour-extinction relation.

Secondly, we can study the colour distribution of these samples. Figure 8 shows the lower part of the histogram in colour $(J-K)^{\mathrm{o}, \mathrm{c}}$, where we applied $f=0.83$ to the extinction correction to be as close to the intrinsic colour as possible. The figure shows various redshift ranges in colour (low to high redshifts going from cyan through green to red, with the full sample depicted in black), together with the galaxies that have no velocity information in dark blue. The median $(J-K)^{\mathrm{o}, \mathrm{c}}$-values go from $0 .{ }^{\mathrm{m}} 981$ through $1^{\mathrm{m}} 034$ to $1^{\mathrm{m}} 054$, respectively, while the no-redshift sample has a median of 1. 044 , albeit with a larger scatter of 0.20 (as compared to $0 .{ }^{\mathrm{m}} 10-0 . \mathrm{m} 16$ for the other samples). Though NIR colours are only a rough indicator of redshift (e.g., Jarrett 2004), this shows that the missing galaxies seem to be well distributed across redshift space with no likely bias in distance (or k-correction).

We thus conclude that though the sub-sample of galaxies with redshifts gives a different $f$-value, the k-correction itself has a negligible effect on its determination: (1) the missing galaxies seem to have a similar redshift distribution to those with redshifts, with a moderate lack of very close-by galaxies $\left(c z \lesssim 3000 \mathrm{~km} \mathrm{~s}^{-1}\right)$, (2) the amount of $\mathrm{k}$ - 
A comparative analysis of Galactic extinction

Table 3. Comparison of parameters for different apertures

\begin{tabular}{lcccccc}
\hline & A5 & A7 & A10 & ISO & S5 & PK \\
\hline$f$-value & 0.832 & 0.827 & 0.829 & 0.826 & 0.823 & 0.828 \\
$f_{\text {min }}$ & 0.825 & 0.822 & 0.824 & 0.821 & 0.815 & 0.823 \\
$f_{\text {max }}$ & 0.832 & 0.830 & 0.836 & 0.835 & 0.827 & 0.831 \\
Scatter & $0^{\mathrm{m}} \cdot 141$ & $0^{\mathrm{m}} 134$ & $0^{\mathrm{m}} 130$ & $0^{\mathrm{m}} 129$ & $0^{\mathrm{m}} 134$ & 0.173 \\
$N$ & 3447 & 3460 & 3395 & 3272 & 3391 & 3391 \\
\hline
\end{tabular}

Note: All values have an error of 0.009 except for PK which has an error of 0.012 .

correction does not show any correlation with extinction for this sample, and (3) the missing galaxies come mainly from the intermediate-to-high extinction range which severely depletes the colour-extinction relation in these areas, likely explaining the deviant $f$-value. In fact, we ought to adjust the upper limit in $A_{\mathrm{K}}$ (as discussed in Sec. 3.6) to about $A_{\mathrm{K}}<1$. 04 (at the end of the new 'plateau'), leading to a slight increase of the $f$-value to $0.809 \pm 0.010$, which deviates by only $2 \sigma$ from the original value.

\subsection{Bootstrapping}

In Sec. 3.7 we investigated the effect of the remaining outliers on the linear regression and found it to be negligible. To look further into the effects of sample selection on the result, we applied the bootstrap method, excluding a third of randomly selected data points. We ran this 20 times and found a mean $f=0.831 \pm 0.002$ with individual values ranging from 0.818 to 0.848 . This agrees well with the $f$-value for our cleaned sample, $f=0.827 \pm 0.009$ and we conclude that our result is robust with respect to sample selection and outliers.

\subsection{Discussion}

In conclusion we can say that our derivation of a correction factor to the SFD maps, $f=0.83 \pm 0.01$, is very robust. The largest variation is found across the colours (see Table 21), which seem to be sensitive to the adopted extinction law. The choice of aperture size has a small effect, with $f$-values ranging from 0.823 (S5) to 0.832 (A5), see Table 3 Additional sample selections (as discussed in Secs 3.5 and 3.7] have a similar impact $(0.815-0.836$ across all apertures, see minimum and maximum values in Table 33). A7 and A5 seem to be the most robust, while ISO and PK seem to be more vulnerable (they also have the least objects in the sample). It is notable that even the isophotal colours, much more subject to insufficient star subtraction, show little deviation and agree well with our final value of $f=0.827 \pm 0.009$. The effects of corrections for radii, stellar densities and $\mathrm{k}$ correction are negligible. In the latter case, the galaxies without redshift ( $13 \%$ of the cleaned sample) cause a bias since mostly higher extinction objects are lacking, resulting in a lower $f$-value of $0.801 \pm 0.010$. The bias is expected to disappear once all galaxies have a redshift measured. The nominal uncertainty on our $f$-value is 0.009 , and, based on the variations found, we do not expect the final error to be higher than 0.01 .

\section{OTHER EXTINCTION MAPS}

The analysis we have shown so far was based on the all-sky extinction map derived from DIRBE/IRAS data by SFD which is the most widely used to date (recently with the SF11 modulation). Recently, various extinction maps based on Planck satellite data have become available. We can now use our cleaned sample to compare their quality and whether they may also need a correction factor. In particular, we investigated the following four maps, all of which have a spatial resolution of 6.1 (i.e., comparable to SFD):

P-PR1 The first Planck extinction map (Planck Collaboration et al. 2014) is based on the PR1 data from 2013 and the IRAS $100 \mu \mathrm{m}$ data. A modified blackbody model was used to fit the data.

P-MF Meisner \& Finkbeiner (2015) also used the Planck PR 1 and DIRBE/IRAS $100 \mu \mathrm{m}$ data. Instead of a modified blackbody model, they used a two-component model. Their map is given in optical depth at a frequency of $545 \mathrm{GHz}$ $(\lambda=550 \mu \mathrm{m})$, with a provided conversion of $E(B-V)=$ 2624.4472 - TAU545 - 0.00260618 .

P-AV Planck Collaboration et al. (2016a) fitted a physical dust model from Draine \& Li (2007) to the full mission Planck PR2 (2015) data, WISE $12 \mu \mathrm{m}$ data and IRAS $60 \mu \mathrm{m}$ as well as $100 \mu \mathrm{m}$ data. Their extinction map is given in $A_{\mathrm{V}}$ (their renormalised $\mathrm{AV} \_\mathrm{RQ}$ ), which we converted to $E(B-V)$ using $R_{\mathrm{V}}=3.1$.

P-GNILC Planck Collaboration et al. (2016b) used the PR2 Planck data in combination with the IRAS $100 \mu$ m map and took special care to reduce the cosmic infrared background contamination in the data, using the generalised needlet internal linear combination (GNILC) componentseparation method. Their map is given in dust optical depth at $353 \mathrm{GHz}(\lambda=849 \mu \mathrm{m})$, with a provided conversion of $E(B-V)=14900 \cdot$ TAU353.

We have not used other available maps because they either have lower spatial resolutions, do not cover the whole Galactic plane (e.g., Nidever et al. 2012), or are 3-dimensional, that is, do not cover the full line-of-sight out of our Galaxy (e.g., Gonzalez et al. 2012).

To compare the Planck maps with the SFD map, we have used the colour-extinction relation (Fig. 9), assuming the scatter to be an indicator of the quality of the extinction values since all other parameters are kept unchanged. As mentioned above, the SFD colour-extinction relation has a scatter of 0.134 . In comparison, the P-PR 1 map is the worst with a considerably larger scatter of 0.175 . The other three maps lead to a similarly reduced scatter between $0{ }^{\mathrm{m}} 116$ and 0. 119 (see Table 4), indicating that both improved methods and improved data are of advantage. This comparison is also valid if we consider only galaxies with $|b| \geq 5^{\circ}$ where the SFD maps are fully calibrated.

Interestingly, all relations show still a considerable dependence on extinction, implying the continued need of a correction factor similar to that for the SFD map: the PGNILC map shows the least dependence on extinction with $f=0.859 \pm 0.008$, while both $\mathrm{P}-\mathrm{AV}$ and $\mathrm{P}-\mathrm{MF}$ show the highest dependence $(f=0.759 \pm 0.007$ and $f=0.760 \pm 0.007$, respectively). The offset (that is, the $(J-K)$-colour at zero extinction) is comparable for all maps except for P-PR1, which is significantly redder at $1.080 \pm 0$. 005 . 


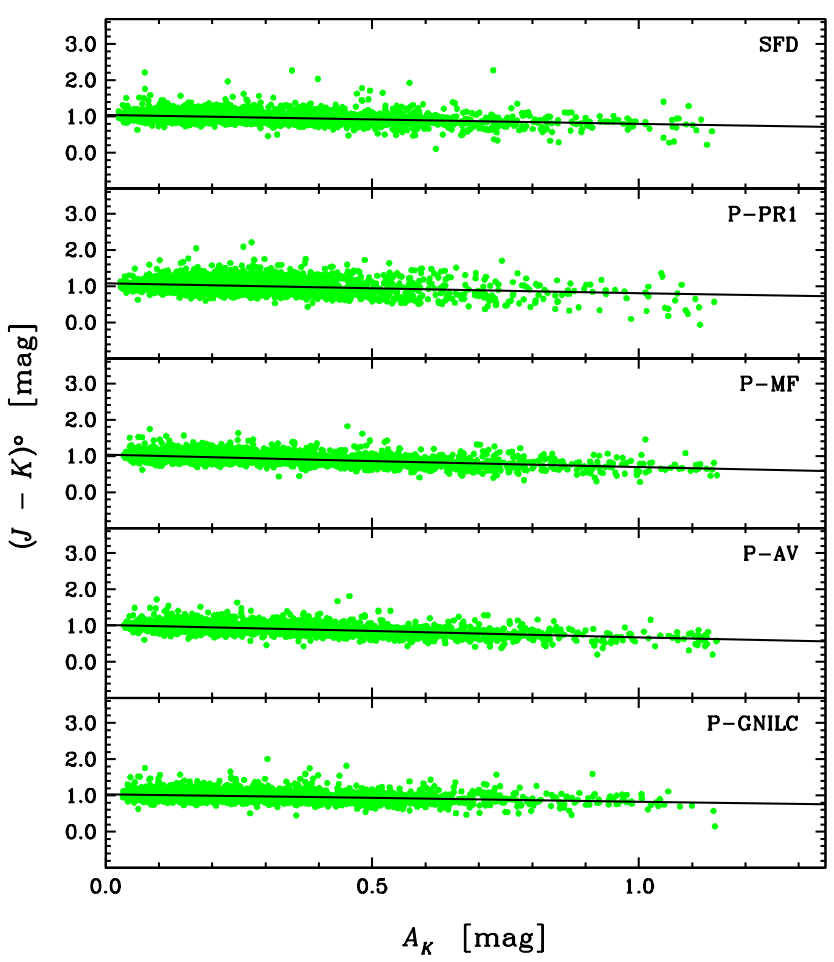

Figure 9. Same as Fig. 1 1 for various extinction maps as indicated by the labels.

Table 4. Comparison of parameters for different extinction maps

\begin{tabular}{lccc}
\hline Extinction map & $f$-value & $\begin{array}{c}\text { scatter } \\
{[\mathrm{mag}]}\end{array}$ & $\begin{array}{c}\text { offset } \\
{[\mathrm{mag}]}\end{array}$ \\
\hline SFD & $0.827 \pm 0.009$ & 0.134 & $1.036 \pm 0.004$ \\
P-PR1 & $0.808 \pm 0.013$ & 0.175 & $1.080 \pm 0.005$ \\
P-MF & $0.760 \pm 0.007$ & 0.118 & $1.031 \pm 0.004$ \\
P-AV & $0.759 \pm 0.007$ & 0.116 & $1.017 \pm 0.004$ \\
P-GNILC & $0.859 \pm 0.008$ & 0.119 & $1.028 \pm 0.004$ \\
\hline
\end{tabular}

We conclude that the three maps P-MF, P-AV and PGNILC are preferable over the SFD map, while the P-PR1 map appears unreliable.

\section{VARIATION ACROSS THE ZOA}

SFD give the caveat that their extinction maps are not calibrated at $|b|<5^{\circ}$, partly because of low galaxy counts, partly because of unsubtracted FIR sources (see discussion in Paper I), and partly because of badly handled artefacts in the temperature map. Our sample is large enough that we can divide the ZoA into smaller cells to look for any largescale variation with both Galactic longitude and latitude. Figure 10 shows the distribution of the sample galaxies in Galactic coordinates (grey dots) with contours of Galactic extinction overlaid as indicated in the caption. The individual cells $\left(60^{\circ} \times 5^{\circ}\right)$ are also shown. It is obvious that some cells have only few galaxies (around the Galactic bulge), while other cells show only little variation in extinction.

To minimise the uncertainties in the slope of the colourextinction relation for the individual cells, in particular where the extinction range is small, we kept the intercept (i.e., the unobscured colour) fixed at the value derived for the full sample. We then determined the $f$-value for each cell, keeping an eye on errors, scatter and extinction range and using the bootstrap method to further estimate the reliability. To avoid the above mentioned problems of the small cell size, we also binned up cells in various ways to search for reliable patterns.

We find that the $f$-value varies across the ZoA between $0.70 \pm 0.04$ and $0.91 \pm 0.03$ or, in more useful terms, the deviation $\Delta f$ from the full sample $f$-value $\left(f_{\text {cell }}-f_{\text {full }}\right)$ goes from -0.13 to 0.08 . We have visualised the results in Fig. 11 by using a colour map of the cells from dark green (most negative) to red (most positive). Numbers in red denote a short extinction range (with a maximum of $A_{\mathrm{K}}=0.55$ ) to indicate that the $f$-value is possibly overestimated (see Fig 5). Values with a colon are deemed less reliable due to larger errors, small number counts or large variation when using the bootstrap method. Despite these problems there appears to be a pattern, mainly along Galactic longitudes. Hence, we have binned the cells to emphasise the Galactic Centre region $\left(-60^{\circ}<l<+60^{\circ}\right)$ versus the Anticentre region $\left(120^{\circ}<l<240^{\circ}\right)$ as shown in the lower panels of the figure. It is now apparent that the $f$-value towards the Galactic Centre is somewhat lower than towards the Anticentre region (by about 0.06 or $6 \sigma$ when binning over all latitudes). A possible variation with latitude is less clear and subject to higher uncertainties in the lowest latitude bin $\left(-10^{\circ}<b<-5^{\circ}\right)$. We also looked for a possible variation with the Cosmic Microwave Background (CMB) dipole and used $120^{\circ}$-wide latitude bins around $l=270^{\circ}$ and $l=90^{\circ}$. The differences here are negligible and reveal no clear pattern.

\subsection{Comparative analysis of the available extinction maps}

We have applied the same analysis to the other extinction maps to see whether they also reveal a variation with position in the sky. For easier comparison, Figs 12 and 13 show histograms of the differences in $f$-values and scatter (green histograms denote the 24 small, independent cells, black stands for all 70 cells used). The individual maps, in the style of Fig. 11 are given in the Appendix.

In the case of P-PR1 we find much larger variations of the $f$-value with more extreme values, varying from 0.24 to $1.24(-0.57 \leq \Delta f \leq 0.43)$. The scatter of the individual colour-extinction relations, on the other hand, is usually much smaller than for the full sample, which, together with the larger variation in slopes, explains the higher scatter of the full relation. Interestingly, the emerging pattern is similar to the SFD map, with the Galactic Centre region having lower $f$-values than the Anticentre region, in this case by the considerable amount of 0.59 . As to variations with latitude, there seems to be a small decrease in the $f$ value in the Centre region from above to below the plane by about 0.18. Considering the large variation from cell to cell, though, this may not be indicative of a coherent pattern, in particular since the other longitude cells do not show a similar change. The cells depicting the $\mathrm{CMB}$ dipole also show a small difference $(\Delta f=0.17)$, but considering the map of the 


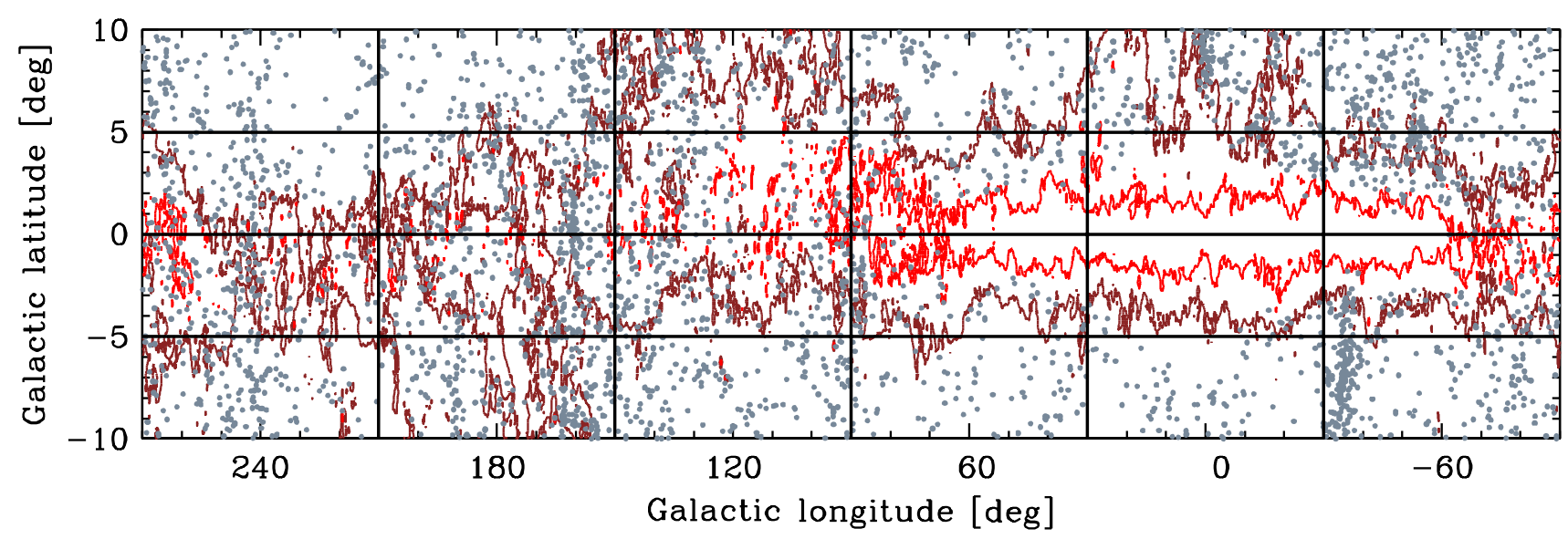

Figure 10. Sky map with contours of Galactic extinction $\left(A_{\mathrm{K}}=0.3\right.$ in brown and $A_{\mathrm{K}}=1$. 0 in red; from SFD). Galaxies are shown as grey dots. The grid depicts the individual cells used in the analysis.

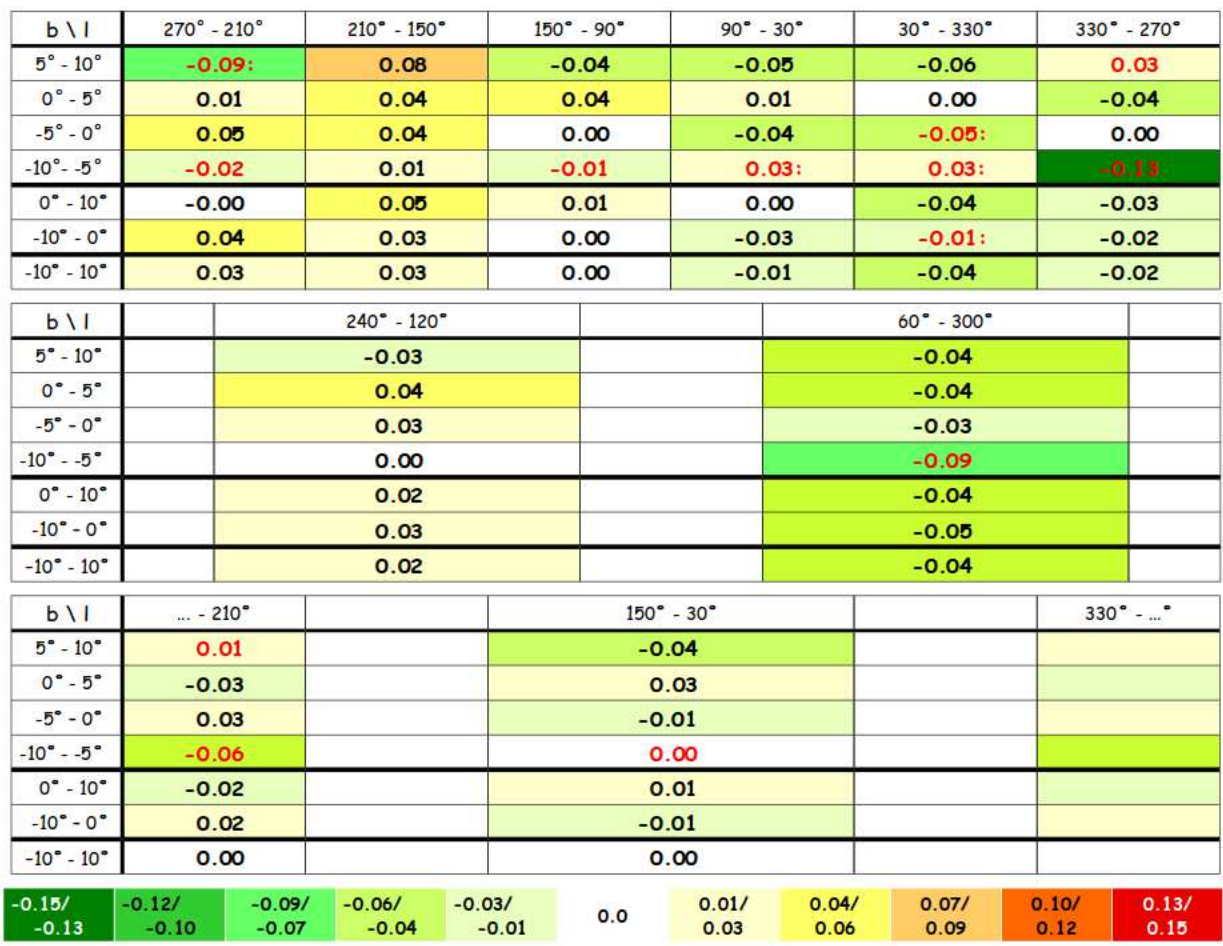

Figure 11. Binned sky map with differential $f$-values $\left(f_{\text {cell }}-f_{\text {full }}\right)$ per cell. The colour scale is shown at the bottom. Red numbers may be overestimated and colons indicate uncertain values. The $330^{\circ}-210^{\circ}$ cells are wrapped around the Galactic Centre.

individual cells this seems only to reflect the much stronger Centre-Anticentre juxtaposition.

The analysis of the improved Planck PR1 map P-MF shows similar variations as SFD and reflects the small scatter of the full sample colour-extinction relation. The $f$-values vary from 0.68 to $0.90(-0.08 \leq \Delta f \leq 0.14)$. Again, there is a dichotomy between the Galactic Centre and Anticentre regions, but, contrary to the SFD maps, the Centre region shows higher values than the Anticentre region, by about 0.05 or $5 \sigma$. As with the SFD maps, there is no clear variation with latitude or the CMB dipole.

The P-AV map, based on Planck PR2 data, shows slightly larger variations than SFD, going from 0.61 to 0.91 $(-0.15 \leq \Delta f \leq 0.15)$. As for SFD, the Galactic Centre region shows lower values than the Anticentre region, but more pronounced with a difference of 0.08 or $8 \sigma$. As for PPR1, there appears to be a possible decrease in the $f$-value with latitude in the Galactic Centre region (by 0.05 or $5 \sigma$ ), but the same caveat applies here: variations from cell to cell are of similar order (albeit with larger errors), and the Anticentre region does not show any pattern. The difference with respect to the $\mathrm{CMB}$ dipole only reflects the stronger Centre-Anticentre juxtaposition.

The P-GNILC map shows the least variations, going from 0.77 to $0.93(-0.09 \leq \Delta f \leq 0.07)$. In this case, no pattern can be discerned. The difference between the Galactic Centre and Anticentre region is $0.01(1 \sigma)$ and $0.03(3 \sigma)$ for 


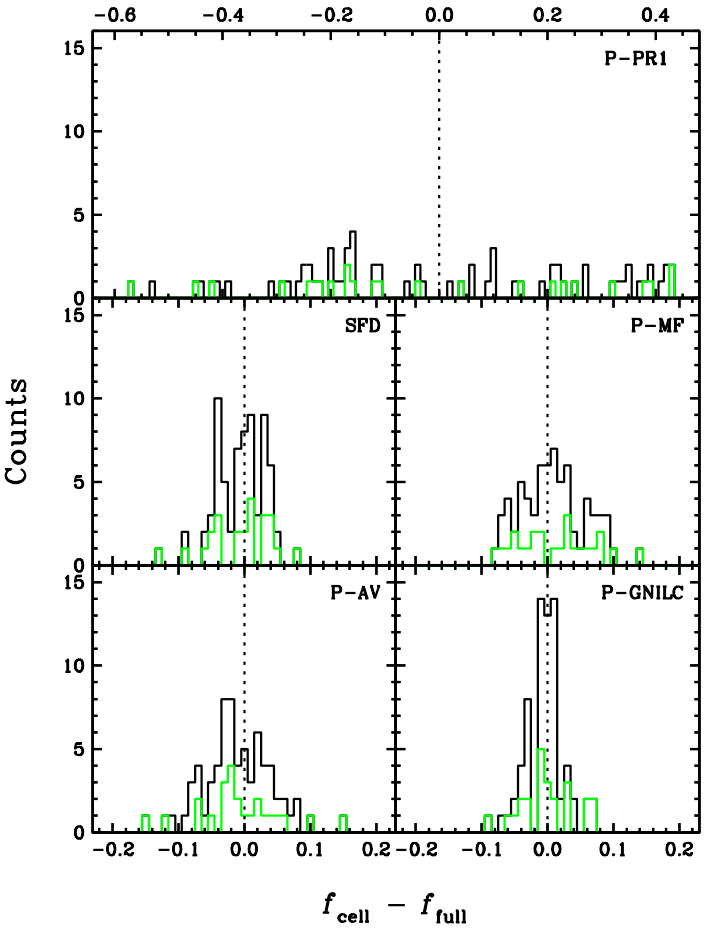

Figure 12. Histograms of $\Delta f$ for all extinction maps, as labelled. Green histograms indicate the individual cells $\left(60^{\circ} \times 5^{\circ}\right)$ and black histograms comprise all cells analysed. Dotted vertical lines indicate $\Delta f=0$.

the CMB dipole. No latitude dependence could be discerned either.

Considering Figs 12 and 13 P-PR1 shows strikingly different behaviour compared to the other maps: there is no preferred value for $f$ at all, instead it varies evenly across the whole spread of values. The values of the scatter of the individual cells, on the other hand, are markedly smaller, confirming that the large scatter of the full sample is either due to varying background contamination or to insufficient calibration anchors across the ZoA. In fact, the other four maps also show a slightly smaller scatter for the individual cells, which likely means that the variation in the $f$-value is real. The $\Delta f$ of these four maps show the more expected grouping around zero, though only the P-GNILC map shows a pronounced peak at zero for both the individual cells as well as for the binned ones. P-AV, in particular, seems to show a bipolar distribution instead.

It is worth noting that for the map with the least variations and smallest correction factor, P-GNILC, particular care was given by the authors to the removal of any residuals due to the anisotropy of the CMB. Based on the overall comparison, we recommend the use of this map in preference to the SFD map.

\section{SUMMARY AND CONCLUSIONS}

In this paper, we have used 2MASS NIR colours of galaxies to calibrate the DIRBE/IRAS extinction map by SFD at low Galactic latitudes. We have derived a correction factor $f=0.83 \pm 0.01$ by fitting a linear regression to the colourextinction relation, using 3460 galaxies.

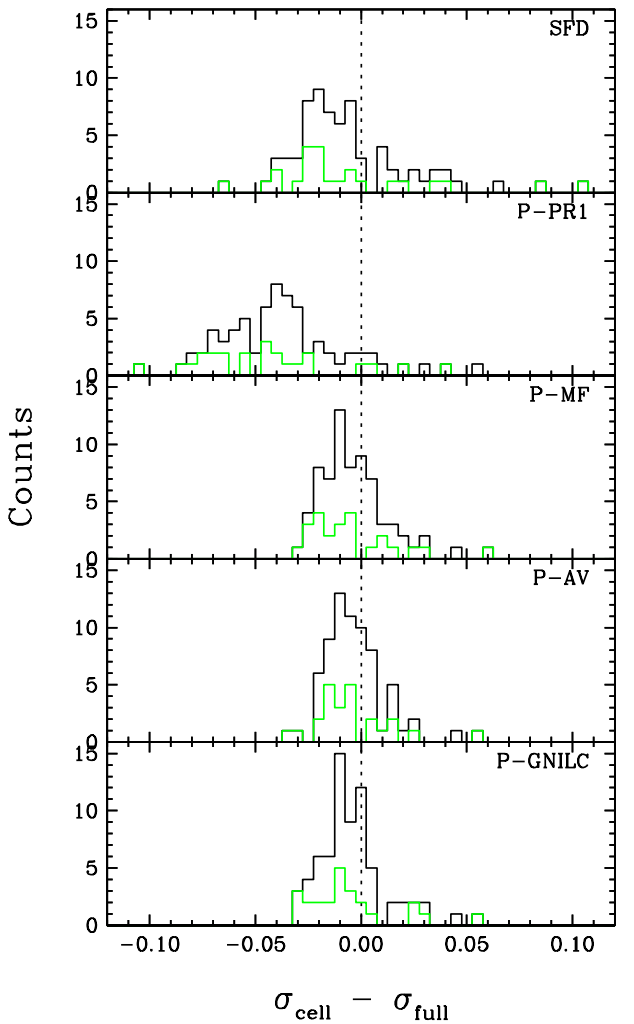

Figure 13. Same as Fig. 12 for the differential scatter in the colour-extinction relation.

In the first part of the paper, we concentrated on finding the best parameter settings and corrections for a clean analysis, using the widely used SFD DIRBE/IRAS maps. We found that the $(J-K)$-colour from the $7^{\prime \prime}$-radius aperture is most useful, balancing the lower photometric uncertainty of a small aperture, little affected by contamination with stars, against the need to retain an as large as possible sample for the subsequent analysis. We therefore applied only the most necessary cuts in photometric errors and flag settings.

One of the problems of a reliable colour-extinction relation is the coarse spatial resolution of the extinction maps which introduces a bias in the finding of galaxies, that is, galaxies are predominantly found in lower extinction pockets while the average extinction of that area is higher. As a consequence, galaxy colours will appear systematically too blue since the extinction correction applied is too high. We found this to be mainly a problem in high extinction areas where the extinction often varies rapidly due to dark clouds. This is confirmed in the colour-extinction relation that shows the galaxy colours deviating from the linear relation at the higher extinction end. We have thus adopted an upper extinction limit of $A_{\mathrm{K}}=1$. 15 (or $E(B-V) \simeq 3.19$ ).

We investigated the effects of using different extinction laws by comparing the $f$-value derived for the three colours $(J-K),(J-H)$ and $(H-K)$. We have found that the F99 extinction law shows better agreement than the law derived by $\mathrm{C} 89$ but note that the coefficient used for the $H$-band could still be improved.

We tested the application of the k-correction to a subsample of the galaxies with redshift information. While the $\mathrm{k}$-correction itself does not have any effect on the $f$-value, 
A comparative analysis of Galactic extinction

the subsample itself leads to a lower $f$-value of $0.80 \pm 0.01$. This is mainly due to a selection bias in redshift measurements such that smaller galaxies at higher extinctions are less likely to have a redshift measurement. We note, though, that since our sample is magnitude limited with $K^{\mathrm{o}} \leq 11^{\mathrm{m}} 25$, it comprises predominantly near-by galaxies and the spread in k-correction for our sample is small.

The derived correction factor $f=0.83 \pm 0.01$ is slightly smaller than the one found by SF11 $(f=0.86)$ for the SFD maps. Since we agree with SF11 on applying the F99 reddening law and using $R_{V}=3.1$, the $3-\sigma$ difference (if real) must have other reasons. We have shown that the restriction to a small extinction range $\left(A_{\mathrm{K}}<0.4\right)$ can lead to slightly higher values with larger uncertainties. SF11 note that they have only little data beyond $A_{\mathrm{K}}=0{ }^{\mathrm{m}} 11$. We thus recommend that at low latitudes $\left(|b|<10^{\circ}\right)$ or high extinctions the application of $f=0.83$ is used instead.

We used our sample to test four other all-sky extinction maps, all based on Planck data. We did not investigate other available maps since they either have a lower spatial resolution, cover only a limited region in the sky or measure extinctions towards stars, which underestimates the full line-of-sight extinction to the extragalactic sky. Next to the original Planck extinction map, based on PR1 data (Planck Collaboration et al. 2014), we used a modified map of the PR1 data by Meisner \& Finkbeiner (2015). Planck PR2 data were used by Planck Collaboration et al. (2016a) and Planck Collaboration et al. (2016b). We found that all maps require a correction factor similar to the one for the SFD maps. Furthermore, the original Planck map (P-PR1) shows a larger scatter and a very high offset in the relation, that is, an unobscured $(J-K)$ colour that is too red.

Our subsequent analysis of a possible variation of the correction factor over Galactic longitude and latitude revealed more problems with the P-PR1 map where the correction factor varies strongly, seemingly even within some of the $60^{\circ} \times 5^{\circ}$ cells. Furthermore we found that all but the P-GNILC map show a small but marked difference between the Galactic Centre and Anticentre regions, and none with the direction of the CMB dipole.

We conclude by recommending the use of the P-GNILC map at low latitudes as showing the least variation across the sky and requiring the smallest correction, $f=0.86 \pm 0.01$.

While our NIR ZoA galaxy sample is the largest and most complete to date, it still suffers from missing galaxies in the highest stellar density region, i.e., the Galactic bulge. With the new NIR large-area surveys UKIDSS-GPS (Lucas et al. 2008) and VVV (e.g., Minniti et al. 2010) we can fill in the gap and increase the sample size, which will allow a finer binning and thus a more smoothly varying correction factor with longitude.

We also plan to compare in detail the full-sky maps with regional 3D maps and to investigate ways to calibrate the 3D maps to the full line-of-sight extinctions displayed in the coarser maps while retaining the higher spatial resolution information. This may only be possible, though, where stars at the far side of the Galactic Bulge were used.

\section{ACKNOWLEDGMENTS}

The authors thank the observing teams in our project for their efforts and for making spectroscopic data available prior to publications: L. Macri, T. Lambert, K. Said. This publication makes use of data products from the Two Micron All Sky Survey, which is a joint project of the University of Massachusetts and the Infrared Processing and Analysis Center, funded by the National Aeronautics and Space Administration and the National Science Foundation. This research also has made use of the HyperLeda database, the SIMBAD database, operated at CDS, Strasbourg, France, the NASA/IPAC Extragalactic Database (NED) which is operated by the Jet Propulsion Laboratory, California Institute of Technology, under contract with the National Aeronautics and Space Administration and the Sloan Digital Sky Survey which is managed by the Astrophysical Research Consortium for the Participating Institutions. RKK's research is supported by the South African Research Chair Initiative of the Department of Science \& Innovation and the National Research Foundation (NRF). ACS thanks the South African NRF for their financial support.

\section{DATA AVAILABILITY}

The data underlying this article are available in the article and in its online supplementary material.

\section{REFERENCES}

Acker A., Stenholm B., Veron P., 1991, A\&AS, 87, 499

Alam S., Albareti F. D., Allende Prieto C., Anders F., Anderson S. F., Anderton T., Andrews B. H., Armengaud E., Aubourg É., et al., 2015, ApJS, 219, 12

Ali A., Dopita M. A., Basurah H. M., Amer M. A., Alsulami R., Alruhaili A., 2016, MNRAS, 462, 1393

Beaulieu S. F., Dopita M. A., Freeman K. C., 1999, ApJ, 515,610

Beuing J., Bender R., Mendes de Oliveira C., Thomas D., Maraston C., 2002, A\&A, 395, 431

Bicay M. D., Giovanelli R., 1986a, AJ, 91, 705

Bicay M. D., Giovanelli R., 1986b, AJ, 91, 732

Bicay M. D., Giovanelli R., 1987, AJ, 93, 1326

Bikmaev I. F., Burenin R. A., Revnivtsev M. G., Sazonov S. Y., Sunyaev R. A., Pavlinsky M. N., Sakhibullin N. A., 2008, Astronomy Letters, 34, 653

Bottinelli L., Durand N., Fouque P., Garnier R., Gouguenheim L., Paturel G., Teerikorpi P., 1992, A\&AS, 93, 173

Bottinelli L., Durand N., Fouque P., Garnier R., Gouguenheiml. Loulergue M., Paturel G., Petit C., Teerikorpi P., 1993, A\&AS, 102, 57

Bottinelli L., Gouguenheim L., Loulergue M., Martin J. M., Theureau G., Paturel G., 1994, in Balkowski C., KraanKorteweg R. C., eds, Unveiling Large-Scale Structures Behind the Milky Way Vol. 67 of Astronomical Society of the Pacific Conference Series, An HI-Search for IRAS Galaxies in the Galactic Plane. p. 225

Bottinelli L., Gouguenheim L., Paturel G., 1981, A\&AS, 44, 217

Bronfman L., Nyman L. A., May J., 1996, A\&AS, 115, 81

Burbidge E. M., Burbidge G. R., 1972, ApJ, 172, 37 
Burenin R. A., Mescheryakov A. V., Revnivtsev M. G., Sazonov S. Y., Bikmaev I. F., Pavlinsky M. N., Sunyaev R. A., 2008, Astronomy Letters, 34, 367

Burstein D., Davies R. L., Dressler A., Faber S. M., Stone R. P. S., Lynden-Bell D., Terlevich R. J., Wegner G., 1987, ApJS, 64, 601

Burstein D., Heiles C., 1978, ApJ, 225, 40

Burstein D., Heiles C., 1982, AJ, 87, 1165

Cardelli J. A., Clayton G. C., Mathis J. S., 1989, ApJ, 345, 245

Chamaraux P., Cayatte V., Balkowski C., Fontanelli P., 1990, A\&A, 229, 340

Chamaraux P., Masnou J.-L., Kazés I., Saitō M., Takata T., Yamada T., 1999, MNRAS, 307, 236

Chiang Y.-K., Ménard B., 2019, ApJ, 870, 120

Chilingarian I. V., Melchior A.-L., Zolotukhin I. Y., 2010, MNRAS, 405, 1409

Choloniewski J., Valentijn E. A., 2003, Acta Astron., 53, 265

Collobert M., Sarzi M., Davies R. L., Kuntschner H., Colless M., 2006, MNRAS, 370, 1213

Corwin H. G. J., Emerson D., 1982, MNRAS, 200, 621

Cottaar M., Covey K. R., Foster J. B., Meyer M. R., Tan J. C., Nidever D. L., Chojnowski S. D., da Rio N., Flaherty K. M., Frinchaboy P. M., Majewski S., Skrutskie M. F., Wilson J. C., Zasowski G., 2015, ApJ, 807, 27

Courtois H. M., Tully R. B., 2015, MNRAS, 447, 1531

Courtois H. M., Tully R. B., Fisher J. R., Bonhomme N., Zavodny M., Barnes A., 2009, AJ, 138, 1938

Courtois H. M., Tully R. B., Makarov D. I., Mitronova S., Koribalski B., Karachentsev I. D., Fisher J. R., 2011, MNRAS, 414, 2005

Crook A. C., Huchra J. P., Martimbeau N., Masters K. L., Jarrett T., Macri L. M., 2007, ApJ, 655, 790

Crook A. C., Huchra J. P., Martimbeau N., Masters K. L., Jarrett T., Macri L. M., 2008, ApJ, 685, 1320

Danziger I. J., Goss W. M., 1983, MNRAS, 202, 703

Davies R. D., Staveley-Smith L., Murray J. D., 1989, MNRAS, 236, 171

Davies R. L., Burstein D., Dressler A., Faber S. M., Lynden-Bell D., Terlevich R. J., Wegner G., 1987, ApJS, 64,581

Davoust E., Considere S., 1995, A\&AS, 110, 19

de Vaucouleurs G., de Vaucouleurs A., Corwin Herold G. J., Buta R. J., Paturel G., Fouque P., 1991, Third Reference Catalogue of Bright Galaxies

Demoulin M.-H., 1970, ApJ, 160, L79

di Nella H., Couch W. J., Parker Q. A., Paturel G., 1997, MNRAS, 287, 472

di Nella H., Paturel G., Walsh A. J., Bottinelli L., Gouguenheim L., Theureau G., 1996, A\&AS, 118, 311

Djorgovski S., Thompson D. J., de Carvalho R. R., Mould J. R., 1990, AJ, 100, 599

Donley J. L., Koribalski B. S., Staveley-Smith L., KraanKorteweg R. C., Schröder A., Henning P. A., 2006, MNRAS, 369, 1741

Donley J. L., Staveley-Smith L., Kraan-Korteweg R. C., Islas-Islas J. M., Schröder A., Henning P. A., Koribalski B., Mader S., Stewart I., 2005, AJ, 129, 220

Doyle M. T., Drinkwater M. J., Rohde D. J., Pimbblet K. A., Read M., Meyer M. J., Zwaan M. A., Ryan-Weber E., Stevens J., Koribalski B. S., et al., 2005, MNRAS, 361,
34

Draine B. T., Li A., 2007, ApJ, 657, 810

Dressler A., 1991, ApJS, 75, 241

Durret F., Wakamatsu K., Nagayama T., Adami C., Biviano A., 2015, A\&A, 583, A124

Dutra C. M., Ahumada A. V., Clariá J. J., Bica E., Barbuy B., 2003, A\&A, 408, 287

Evans C. J., Kennedy M. B., Dufton P. L., Howarth I. D., Walborn N. R., Markova N., Clark J. S., de Mink S. E., de Koter A., et al., 2015, A\&A, 574, A13

Fairall A. P., 1981, MNRAS, 196, 417

Fairall A. P., 1983, MNRAS, 203, 47

Fairall A. P., 1988a, MNRAS, 230, 69

Fairall A. P., 1988b, MNRAS, 233, 691

Fairall A. P., Vettolani G., Chincarini G., 1989, A\&AS, 78, 269

Fairall A. P., Woudt P. A., 2006, MNRAS, 366, 267

Fairall A. P., Woudt P. A., Kraan-Korteweg R. C., 1998, A\&AS, 127, 463

Falco E. E., Kurtz M. J., Geller M. J., Huchra J. P., Peters J., Berlind P., Mink D. J., Tokarz S. P., Elwell B., 1999, PASP, 111, 438

Fürész G., Hartmann L. W., Megeath S. T., Szentgyorgyi A. H., Hamden E. T., 2008, ApJ, 676, 1109

Fisher J. R., Tully R. B., 1981, ApJS, 47, 139

Fisher K. B., Huchra J. P., Strauss M. A., Davis M., Yahil A., Schlegel D., 1995, ApJS, 100, 69

Fitzpatrick E. L., 1999, PASP, 111, 63

Foley R. J., 2009, Central Bureau Electronic Telegrams, 1855, 1

Foster J. B., Cottaar M., Covey K. R., Arce H. G., Meyer M. R., Nidever D. L., Stassun K. G., Tan J. C., Chojnowski S. D., da Rio N., Flaherty K. M., Rebull L., Frinchaboy P. M., Majewski S. R., Skrutskie M., Wilson J. C., Zasowski G., 2015, ApJ, 799, 136

Fouque P., Durand N., Bottinelli L., Gouguenheim L., Paturel G., 1990, A\&AS, 86, 473

Freeman K. C., Karlsson B., Lynga G., Burrell J. F., van Woerden H., Goss W. M., Mebold U., 1977, A\&A, 55, 445

Freudling W., 1995, A\&AS, 112, 429

Fuerst E., Reich W., Kuehr H., Stickel M., 1989, A\&A, 223, 66

Garrido O., Marcelin M., Amram P., Boissin O., 2003, A\&A, 399, 51

Giovanelli R., Haynes M. P., 1981, AJ, 86, 340

Giovanelli R., Haynes M. P., 1982, AJ, 87, 1355

Goncalves T. S., Martin D. C., Halpern J. P., Eracleous M., Pavlov G. G., 2008, The Astronomer's Telegram, 1623, 1 Gonzalez O. A., Rejkuba M., Zoccali M., Valenti E., Minniti D., Schultheis M., Tobar R., Chen B., 2012, A\&A, 543, A13

Gregory S. A., Tifft W. G., Moody J. W., Newberry M. V., Hall S. M., 2000, AJ, 119, 545

Harris W. E., 1996, AJ, 112, 1487

Hasegawa T., Wakamatsu K.-i., Malkan M., Sekiguchi K., Menzies J. W., Parker Q. A., Jugaku J., Karoji H., Okamura S., 2000, MNRAS, 316, 326

Hauschildt M., 1987, A\&A, 184, 43

Haynes M. P., Giovanelli R., Chamaraux P., da Costa L. N., Freudling W., Salzer J. J., Wegner G., 1999, AJ, 117, 2039 Haynes M. P., Giovanelli R., Herter T., Vogt N. P., Freudling W., Maia M. A. G., Salzer J. J., Wegner G., 
A comparative analysis of Galactic extinction

1997, AJ, 113, 1197

Haynes M. P., Giovanelli R., Starosta B. M., Magri C., 1988, AJ, 95, 607

Haynes M. P., Hogg D. E., Maddalena R. J., Roberts M. S., van Zee L., 1998, AJ, 115, 62

Henning P. A., 1992, ApJS, 78, 365

Henning P. A., Springob C. M., Minchin R. F., Momjian E., Catinella B., McIntyre T., Day F., Muller E., Koribalski B., Rosenberg J. L., Schneider S., Staveley-Smith L., van Driel W., 2010, AJ, 139, 2130

Hill G. J., Heasley J. N., Becklin E. E., Wynn-Williams C. G., 1988, AJ, 95, 1031

Hong T., Staveley-Smith L., Masters K. L., Springob C. M., Macri L. M., Koribalski B. S., Jones D. H., Jarrett T. H., Crook A. C., 2013, MNRAS, 432, 1178

Huchra J., Davis M., Latham D., Tonry J., 1983, ApJS, 52, 89

Huchra J., Geller M., Clemens C., Tokarz S., Michel A., 1995

Huchra J. P., Macri L. M., Masters K. L., Jarrett T. H., Berlind P., Calkins M., 2012, ApJS, 199, 26

Huchra J. P., Macri L. M., Masters K. L., Jarrett T. H., Berlind P., Calkins M., Crook A. C., Cutri R., Erdoğdu P., et al., 2012, ApJS, 199, 26

Huchra J. P., Vogeley M. S., Geller M. J., 1999, ApJS, 121, 287

Huchtmeier W. K., Karachentsev I. D., Karachentseva V. E., 2001, A\&A, 377, 801

Huchtmeier W. K., Karachentsev I. D., Karachentseva V. E., 2003, A\&A, 401, 483

Huchtmeier W. K., Karachentsev I. D., Karachentseva

V. E., Ehle M., 2000, A\&AS, 141, 469

Huchtmeier W. K., Karachentsev I. D., Karachentseva V. E., Kudrya Y. N., Mitronova S. N., 2005, A\&A, 435, 459

Huchtmeier W. K., Lercher G., Seeberger R., Saurer W., Weinberger R., 1995, A\&A, 293, L33

Huchtmeier W. K., Richter O. G., 1989, A General Catalog of HI Observations of Galaxies. The Reference Catalog.

Humason M. L., Mayall N. U., Sandage A. R., 1956, AJ, 61,97

Humason M. L., Wahlquist H. D., 1955, AJ, 60, 254

Im M., Lee I., Cho Y., Choi C., Ko J., Song M., 2007, ApJ, 664,64

Jarrett T. H., 2004, Publications of the Astronomical Society of Australia, 21, 396

Jarrett T. H., Chester T., Cutri R., Schneider S., Rosenberg J., Huchra J. P., Mader J., 2000, AJ, 120, 298

Jones D. H., Read M. A., Saunders W., Colless M., Jarrett T., Parker Q. A., Fairall A. P., Mauch T., Sadler E. M., et al., 2009, MNRAS, 399, 683

Jones D. H., Saunders W., Read M., Colless M., 2005, PASA, 22, 277

Jones P. A., McAdam W. B., 1992, ApJS, 80, 137

Juraszek S. J., Staveley-Smith L., Kraan-Korteweg R. C., Green A. J., Ekers R. D., Haynes R. F., Henning P. A., Kesteven M. J., Koribalski B., Price R. M., Sadler E. M., Schröder A., 2000, AJ, 119, 1627

Karachentsev I. D., 1980, ApJS, 44, 137

Karachentseva V. E., Mitronova S. N., Melnyk O. V., Karachentsev I. D., 2010, Astrophysical Bulletin, 65, 1

Kerr F. J., Henning P. A., 1987, ApJ, 320, L99
Kharchenko N. V., Piskunov A. E., Schilbach E., Röser S., Scholz R. D., 2013, A\&A, 558, A53

Kilborn V. A., Webster R. L., Staveley-Smith L., Marquarding M., Banks G. D., Barnes D. G., Bhathal R., de Blok W. J. G., Boyce P. J., et al., 2002, AJ, 124, 690

Kirhakos S. D., Steiner J. E., 1990, AJ, 99, 1722

Kobulnicky H. A., Dickey J. M., Sargent A. I., Hogg D. E., Conti P. S., 1995, AJ, 110, 116

Koribalski B. S., Staveley-Smith L., Kilborn V. A., Ryder S. D., Kraan-Korteweg R. C., Ryan-Weber E. V., Ekers R. D., Jerjen H., Henning P. A., et al., 2004, AJ, 128, 16 Kraan-Korteweg R. C., Fairall A. P., Balkowski C., 1995, A\&A, 297, 617

Kraan-Korteweg R. C., Henning P. A., Schröder A. C., 2002, A\&A, 391, 887

Kraan-Korteweg R. C., Huchtmeier W. K., 1992, A\&A, 266, 150

Kraan-Korteweg R. C., van Driel W., Schröder A. C., Ramatsoku M., Henning P. A., 2018, MNRAS, 481, 1262

Kregel M., van der Kruit P. C., de Blok W. J. G., 2004, MNRAS, 352, 768

Lahav O., Brosch N., Goldberg E., Hau G. K. T., KraanKorteweg R. C., Loan A. J., 1998, MNRAS, 299, 24

Lang R. H., Boyce P. J., Kilborn V. A., Minchin R. F., Disney M. J., Jordan C. A., Grossi M., Garcia D. A., Freeman K. C., Phillipps S., Wright A. E., 2003, MNRAS, 342, 738

Lara L., Márquez I., Cotton W. D., Feretti L., Giovannini G., Marcaide J. M., Venturi T., 2001, A\&A, 378, 826

Lavaux G., Hudson M. J., 2011, MNRAS, 416, 2840

Lawrence A., Rowan-Robinson M., Ellis R. S., Frenk C. S., Efstathiou G., Kaiser N., Saunders W., Parry I. R., Xiaoyang X., Crawford J., 1999, MNRAS, 308, 897

Lenz D., Hensley B. S., Doré O., 2017, ApJ, 846, 38

Lewis B. M., 1983, AJ, 88, 962

Lewis B. M., 1987, ApJS, 63, 515

Lewis B. M., Davies R. D., 1973, MNRAS, 165, 213

Lewis B. M., Helou G., Salpeter E. E., 1985, ApJS, 59, 161

Liu X.-W., Zhao G., Hou J.-L., 2015, Research in Astronomy and Astrophysics, 15, 1089

Longmore A. J., Hawarden T. G., Goss W. M., Mebold U., Webster B. L., 1982, MNRAS, 200, 325

Lu N. Y., Dow M. W., Houck J. R., Salpeter E. E., Lewis B. M., 1990, ApJ, 357, 388

Lucas P. W., Hoare M. G., Longmore A., Schröder A. C., Davis C. J., Adamson A., Bandyopadhyay R. M., de Grijs R., Smith M., Gosling A., Mitchison S., Gáspár A., Coe M., Tamura M., Parker Q., et al., 2008, MNRAS, 391, 136 Macri L. M., Kraan-Korteweg R. C., Lambert T., Alonso M. V., Berlind P., Calkins M., Erdoğdu P., Falco E. E., Jarrett T. H., Mink J. D., 2019, ApJS, 245, 6

Makarov D. I., Karachentsev I. D., Burenkov A. N., 2003, A\&A, 405, 951

Makarov D. I., Karachentsev I. D., Burennkov A. N., Tyurina N. V., Korotkova G. G., 1997, Astronomy Letters, 23, 638

Martin J. M., Bottinelli L., Dennefeld M., Fouque P., Gouguenheim L., Paturel G., 1990, A\&A, 235, 41

Martin J. M., Bottinelli L., Dennefeld M., Gouguenheim L., 1991, A\&A, 245, 393

Marzke R. O., Huchra J. P., Geller M. J., 1996, AJ, 112, 1803 
Masetti N., Mason E., Morelli L., Cellone S. A., McBride V. A., Palazzi E., Bassani L., Bazzano A., Bird A. J., Charles P. A., Dean A. J., Galaz G., et al., 2008, A\&A, 482, 113

Masetti N., Morelli L., Palazzi E., Galaz G., Bassani L., Bazzano A., Bird A. J., Dean A. J., Israel G. L., Landi R., Malizia A., Minniti D., et al., 2006, A\&A, 459, 21

Masetti N., Parisi P., Palazzi E., Jiménez-Bailón E., Chavushyan V., McBride V., Rojas A. F., Steward L., Bassani L., Bazzano A., et al., 2013, A\&A, 556, A120

Masters K. L., Crook A., Hong T., Jarrett T. H., Koribalski B. S., Macri L., Springob C. M., Staveley-Smith L., 2014, MNRAS, 443, 1044

Mathewson D. S., Ford V. L., 1996, ApJS, 107, 97

Mauch T., Sadler E. M., 2007, MNRAS, 375, 931

McGaugh S. S., Rubin V. C., de Blok W. J. G., 2001, AJ, 122, 2381

McIntyre T. P., Henning P. A., Minchin R. F., Momjian E., Butcher Z., 2015, AJ, 150, 28

Meisner A. M., Finkbeiner D. P., 2015, ApJ, 798, 88

Meyer M. J., Zwaan M. A., Webster R. L., Staveley-Smith L., Ryan-Weber E., Drinkwater M. J., Barnes D. G., Howlett M., Kilborn V. A., et al., 2004, MNRAS, 350, 1195

Michel A., Huchra J., 1988, PASP, 100, 1423

Minniti D., Lucas P. W., Emerson J. P., Saito R. K., Hempel M., Pietrukowicz P., Ahumada A. V., Alonso M. V., Alonso-Garcia J., Arias J. I., Bandyopadhyay R. M., Barbá R. H., Barbuy B., Bedin L. R., Bica E., Borissova J., Bronfman L., et al., 2010, New Astronomy, 15,433

Mitronova S. N., Huchtmeier W. K., Karachentsev I. D., Karachentseva V. E., Kudrya Y. N., 2005, Astronomy Letters, 31, 501

Morganti R., Emonts B., Oosterloo T., 2009, A\&A, 496, L9

Mould J. R., Staveley-Smith L., Schommer R. A., Bothun G. D., Hall P. J., Han M. S., Huchra J. P., Roth J., Walsh W., Wright A. E., 1991, ApJ, 383, 467

Nagayama T., Woudt P. A., Nagashima C., Nakajima Y., Kato D., Kurita M., Nagata T., Nakaya H., Tamura M., Sugitani K., Wakamatsu K., Sato S., 2004, MNRAS, 354, 980

Nakanishi K., Takata T., Yamada T., Takeuchi T. T., Shiroya R., Miyazawa M., Watanabe S., Saitō M., 1997, ApJS, 112, 245

Nataf D. M., Gould A., Fouqué P., Gonzalez O. A., Johnson J. A., Skowron J., Udalski A., Szymański M. K., Kubiak M., Pietrzyński G., Soszyński I., Ulaczyk K., Wyrzykowski Ł., Poleski R., 2013, ApJ, 769, 88

Nidever D. L., Zasowski G., Majewski S. R., 2012, ApJS, 201, 35

Ogando R. L. C., Maia M. A. G., Pellegrini P. S., da Costa L. N., 2008, AJ, 135, 2424

Oh S.-H., Hunter D. A., Brinks E., Elmegreen B. G., Schruba A., Walter F., Rupen M. P., Young L. M., Simpson C. E., Johnson M. C., Herrmann K. A., Ficut-Vicas D., Cigan P., Heesen V., Ashley T., Zhang H.-X., 2015, AJ, 149, 180

Owen F. N., Ledlow M. J., Morrison G. E., Hill J. M., 1997, ApJ, 488, L15

Pantoja C. A., Altschuler D. R., Giovanardi C., Giovanelli
R., 1997, AJ, 113, 905

Pantoja C. A., Giovanardi C., Altschuler D. R., Giovanelli R., 1994, AJ, 108, 921

Parisi P., Masetti N., Jiménez-Bailón E., Chavushyan V., Malizia A., Landi R., Molina M., Fiocchi M., Palazzi E., Bassani L., Bazzano A., et al., 2009, A\&A, 507, 1345

Paturel G., Theureau G., Bottinelli L., Gouguenheim L., Coudreau-Durand N., Hallet N., Petit C., 2003, A\&A, 412, 57

Perlman E. S., Stocke J. T., Schachter J. F., Elvis M., Ellingson E., Urry C. M., Potter M., Impey C. D., Kolchinsky P., 1996, ApJS, 104, 251

Pfleiderer J., Gruber M. D., Gruber G. M., Velden L., 1981, A\&A, 102, L21

Planck Collaboration Abergel A., Ade P. A. R., Aghanim N., Alves M. I. R., Aniano G., Armitage-Caplan C., Arnaud M., Ashdown M., Atrio-Barand ela F., Aumont J., Baccigalupi C., et al., 2014, A\&A, 571, A11

Planck Collaboration Ade P. A. R., Aghanim N., Alves M. I. R., Aniano G., Arnaud M., Ashdown M., Aumont J., Baccigalupi C., Banday A. J., et al., 2016, A\&A, 586, A132

Planck Collaboration Aghanim N., Ashdown M., Aumont

J., Baccigalupi C., Ballardini M., Band ay A. J., Barreiro

R. B., Bartolo N., et al., 2016, A\&A, 596, A109

Radburn-Smith D. J., Lucey J. R., Woudt P. A., Kraan-

Korteweg R. C., Watson F. G., 2006, MNRAS, 369, 1131

Reif K., Mebold U., Goss W. M., van Woerden H., Siegman B., 1982, A\&AS, 50, 451

Richter O.-G., Huchtmeier W. K., 1991, A\&AS, 87, 425

Roman A. T., Takeuchi T. T., Nakanishi K., Saito M., 1998, PASJ, 50, 47

Rubin V. C., Ford W. K. J., Thonnard N., Roberts M. S., Graham J. A., 1976, AJ, 81, 687

Sabbadin F., Cappellaro E., Salvadori L., Turatto M., 1989, ApJ, 347, L5

Sadler E. M., 1984, AJ, 89, 23

Sanchez-Barrantes M., Henning P. A., McIntyre T., Momjian E., Minchin R., Rosenberg J. L., Schneider S., Staveley-Smith L., van Driel W., Ramatsoku M., Butcher Z., Vaez E., 2019, AJ, 158, 234

Sandage A., 1976, PASP, 88, 367

Sanders D. B., Egami E., Lipari S., Mirabel I. F., Soifer B. T., 1995, AJ, 110, 1993

Saunders W., Sutherland W. J., Maddox S. J., Keeble O., Oliver S. J., Rowan-Robinson M., McMahon R. G., Efstathiou G. P., Tadros H., White S. D. M., Frenk C. S., Carramiñana A., Hawkins M. R. S., 2000, MNRAS, 317, 55

Schlafly E. F., Finkbeiner D. P., 2011, ApJ, 737, 103

Schlegel D. J., Finkbeiner D. P., Davis M., 1998, ApJ, 500, 525

Schneider S. E., Thuan T. X., Mangum J. G., Miller J., 1992, ApJS, 81, 5

Schröder A. C., Flöer L., Winkel B., Kerp J., 2019, MNRAS, 489, 2907

Schröder A. C., Kraan-Korteweg R. C., Henning P. A., 2009, A\&A, 505, 1049

Schröder A. C., Mamon G. A., Kraan-Korteweg R. C., Woudt P. A., 2007, A\&A, 466, 481

Schröder A. C., van Driel W., Kraan-Korteweg R. C., 2019, MNRAS, 482, 5167 
A comparative analysis of Galactic extinction

Seeberger R., Huchtmeier W. K., Weinberger R., 1994, A\&A, 286, 17

Seeberger R., Saurer W., 1998, A\&AS, 127, 101

Sekiguchi K., Wolstencroft R. D., 1992, MNRAS, 255, 581

Skrutskie M. F., Cutri R. M., Stiening R., Weinberg M. D., Schneider S., Carpenter J. M., Beichman C., Capps R., Chester T., Elias J., et al., 2006, AJ, 131, 1163

Spinrad H., 1975, ApJ, 199, L1

Springob C. M., Haynes M. P., Giovanelli R., Kent B. R., 2005, ApJS, 160, 149

Staveley-Smith L., Kraan-Korteweg R. C., Schröder A. C., Henning P. A., Koribalski B. S., Stewart I. M., Heald G., 2016, AJ, 151, 52

Stickel M., Kuehr H., Fried J. W., 1993, A\&AS, 97, 483

Stickel M., Lemke D., Klaas U., Krause O., Egner S., 2004, A\&A, 422, 39

Strauss M. A., Huchra J. P., Davis M., Yahil A., Fisher K. B., Tonry J., 1992, ApJS, 83, 29

Sulentic J. W., Arp H., 1983, AJ, 88, 489

Takata T., Yamada T., Saito M., Chamaraux P., Kazes I., 1994, A\&AS, 104, 529

Theureau G., Bottinelli L., Coudreau-Durand N., Gouguenheim L., Hallet N., Loulergue M., Paturel G., Teerikorpi P., 1998, A\&AS, 130, 333

Theureau G., Coudreau N., Hallet N., Hanski M., Alsac L., Bottinelli L., Gouguenheim L., Martin J. M., Paturel G., 2005, A\&A, 430, 373

Theureau G., Coudreau N., Hallet N., Hanski M. O., Poulain M., 2017, A\&A, 599, A104

Theureau G., Hanski M. O., Coudreau N., Hallet N., Martin J. M., 2007, A\&A, 465, 71

Tifft W. G., Cocke W. J., 1988, ApJS, 67, 1

Tobin J. J., Hartmann L., Fűrész G., Hsu W.-H., Mateo M., 2015, AJ, 149, 119

Tully R. B., Fisher J. R., 1988, Catalog of Nearby Galaxies Turatto M., Cappellaro E., Sabbadin F., Salvadori L., 1993, AJ, 105, 142

van den Bosch R. C. E., Gebhardt K., Gültekin K., Yıldırım A., Walsh J. L., 2015, ApJS, 218, 10

van Driel W., Schneider S. E., Kraan-Korteweg R. C., Monnier Ragaigne D., 2009, A\&A, 505, 29

Visvanathan N., van den Bergh S., 1992, AJ, 103, 1057

Visvanathan N., Yamada T., 1996, ApJS, 107, 521

Wegner G., Bernardi M., Willmer C. N. A., da Costa L. N., Alonso M. V., Pellegrini P. S., Maia M. A. G., Chaves O. L., Rité C., 2003, AJ, 126, 2268

West R. M., Surdej J., Schuster H. E., Muller A. B., Laustsen S., Borchkhadze T. M., 1981, A\&AS, 46, 57

West R. M., Tarenghi M., 1989, A\&A, 223, 61

Whiteoak J. B., Gardner F. F., 1977, Australian Journal of Physics, 30, 187

Wilson R. E., 1953, Carnegie Institute Washington D.C. Publication, p. 0

Wong O. I., Ryan-Weber E. V., Garcia-Appadoo D. A., Webster R. L., Staveley-Smith L., Zwaan M. A., Meyer M. J., Barnes D. G., Kilborn V. A., et al., 2006, MNRAS, 371,1855

Woudt P. A., Kraan-Korteweg R. C., Cayatte V., Balkowski C., Felenbok P., 2004, A\&A, 415, 9

Woudt P. A., Kraan-Korteweg R. C., Fairall A. P., 1999, A\&A, 352, 39
Woudt P. A., Kraan-Korteweg R. C., Lucey J., Fairall A. P., Moore S. A. W., 2008, MNRAS, 383, 445

Wouterloot J. G. A., Brand J., 1989, A\&AS, 80, 149

Wynn-Williams C. G., Heasley J. N., Depoy D. L., Hill

G. J., Becklin E. E., 1986, ApJ, 304, 409

Yamada T., Saito M., 1993, PASJ, 45, 25

Yamada T., Tomita A., Saito M., Chamaraux P., Kazes I., 1994, MNRAS, 270, 93

\section{APPENDIX A: CATALOGUE OF VELOCITY MEASUREMENTS AND K-CORRECTIONS}

We searched the online databases HyperLeda, NED and Simbad for velocity information on our objects. In addition, we searched particular publications with the CDS portal for more comprehensive information. Finally, we supplemented the velocity database with the latest data from our on-going observation programmes in the optical and $\mathrm{HI}$, to be published presently. For each measurement that we found we determined whether it was an optical or H I measurement, and whether the reference was original or a compilation. If more than one velocity measurement was found, we chose the best of the optical and the best of the H I measurements where 'best' was usually determined by the error and sometimes by additional information like the velocity resolution of an H I observation. No detailed comparison has been attempted. For our final catalogue, we usually preferred an $\mathrm{H}$ I measurement over an optical for its greater accuracy.

The catalogue of the adopted velocity measurements and k-corrections is available online, where Table A1 lists the first 10 galaxy entries. The table is in the same format as the full object catalogue presented in Paper I with the same ID per row. Some columns are repeated for easy reference. The references for the bibliographic code information used are listed in Table A2 (the full table is available online). The columns of Table A1 are as follows:

Col. 1: ID: 2MASX catalogue identification number (based on J2000.0 coordinates);

Col. 2a and 2b: Galactic coordinates: longitude $l$ and latitude $b$, in degrees;

Col. 3: Extinction: $E(B-V)$ value derived from the DIRBE/IRAS maps (SFD), in mag;

Col. 4: Colour $(J-K)^{\circ}$ corrected for foreground extinction; contrary to the values given in Paper I, these were corrected with the 3 -digit precision extinction coefficients presented in Table 1, in mag;

Col. 5: Sample flag galaxy: 'g' denotes a galaxy (object classes 1-4), and 'p' stands for galaxy candidates (class 5);

Col. 6: Object offset flag: 'o' stands for coordinates that are offset from the centre of the object, and 'e' stands for detections near the edge of an image (at an offset position from the object centre) which were detected with properly centred coordinates on the adjacent image;

Col. 7: Velocity flag for the final adopted velocity: o' stands for an optical measurement and 'o?' indicates a questionable optical measurement or object ID, 'g' the velocity shows it to be a Galactic object, 'h' stands for published H I velocity and 'h:' for uncertain H I velocity, 'h?' is an uncertain object ID (based on a radio telescope's large beam size 
that may include nearby galaxies), and a star indicates a yet unpublished measurement;

Col. 8: 2MRS flag: a star denotes if our adopted velocity, error or reference deviates from the values given in the $2 \mathrm{MRS}$ catalogue (Huchra et al. 2012, Macri et al. 2019);

Col. 9a and 9b: Adopted velocity and error according to the flag in Col. 7 , in $\mathrm{kms}^{-1}$; a star replaces the values in case of unpublished values;

Col. 10: k-correction for the colour $(J-K)$, in mag;

Col. 11a-11c: Adopted optical velocity with error and reference (bibliographic code) for this object, in $\mathrm{kms}^{-1}$; square brackets indicate a discarded measurement; a star replaces the values in case of unpublished measurements;

Col. 12a-12c: Adopted H I velocity with error and reference (bibliographic code) for this object, in $\mathrm{km} \mathrm{s}^{-1}$; square brackets indicate a discarded measurement; a star replaces the values in case of unpublished measurements.

The table lists also some velocity information as yet unpublished. These are identified with a star and are from our observing campaigns: Optical observations in the frame work of the 2MRS/2MZOA surveys are identified by observation year, the ID $2 \mathrm{MRS}$ or $2 \mathrm{MZO}$ and the observatory ID as explained in Macri et al. (2019). H I observations obtained by our group refer to the blind Parkes H I Galactic bulge survey (Kraan-Korteweg et al., in prep.) and to pointed H I observations of or sample with the Parkes telescope (Said, priv.comm.) and the NRT (Kraan-Korteweg et al., in prep.). Finally, some velocities quoted by Simbad do not have a reference. 
Table A2. List of references for velocity measurements; example page, the full table is available online

\begin{tabular}{ll}
\hline Bibcode & Reference \\
\hline 1998A\&AS..130..333T & Theureau et al. (1998) \\
2012ApJS..199..26H & Huchra et al. (2012) \\
2018MNRAS.481.1262K & Kraan-Korteweg et al. (2018) \\
Unpublished optical and H I observations by our team: \\
20182MZO.FLWO.0000M & Macri, priv.comm. \\
\hline
\end{tabular}

Table A1: 2MZOA catalogue with k-correction; example page, the full table is available online

\begin{tabular}{|c|c|c|c|c|c|c|c|c|c|c|c|c|c|c|c|c|c|}
\hline $\begin{array}{l}2 \text { MASX J } \\
\text { (1) }\end{array}$ & $\begin{array}{r}l \\
\text { deg } \\
(2 \mathrm{a})\end{array}$ & $\begin{array}{r}b \\
\text { deg } \\
(2 \mathrm{~b})\end{array}$ & $\begin{array}{r}\text { EBV } \\
\text { mag } \\
(3)\end{array}$ & $\begin{array}{r}(J-K)^{o} \\
\operatorname{mag} \\
(4)\end{array}$ & $\begin{array}{l}\text { gal } \\
\text { (5) }\end{array}$ & $\begin{array}{l}\text { F } \\
\text { off } \\
(6)\end{array}$ & $\begin{array}{l}\text { Flags } \\
\text { vel } \\
(7)\end{array}$ & $2 \mathrm{MRS}$ & $\begin{array}{r}v(\text { adpt }) \\
\mathrm{km} / \mathrm{s} \\
(9 \mathrm{a})\end{array}$ & $\begin{array}{r}v_{\text {err }} \\
\mathrm{km} / \mathrm{s} \\
(9 \mathrm{~b})\end{array}$ & $\begin{array}{r}\text { k-corr } \\
\text { mag } \\
(10)\end{array}$ & $\begin{array}{l}v(\mathrm{op}) \\
\mathrm{km} / \mathrm{s} \\
(11 \mathrm{a})\end{array}$ & $\begin{array}{c}v_{\text {err }} \\
\mathrm{km} / \mathrm{s} \\
(11 \mathrm{~b})\end{array}$ & $\begin{array}{l}\text { Ref } \\
(11 \mathrm{c})\end{array}$ & $\begin{array}{l}v(\mathrm{HI}) \\
\mathrm{km} / \mathrm{s} \\
(12 \mathrm{a})\end{array}$ & $\begin{array}{c}v_{\text {err }} \\
\mathrm{km} / \mathrm{s} \\
(12 \mathrm{~b})\end{array}$ & $\begin{array}{l}\text { Ref } \\
(12 \mathrm{c})\end{array}$ \\
\hline $00000637+5319136$ & 115.217 & -8.780 & 0.300 & 0.99 & $\mathrm{~g}$ & - & o & - & 12447 & 65 & 0.05 & 12447 & 65 & 2012ApJS..199_..26H & - & - & - \\
\hline $00031331+5352149$ & 115.783 & -8.330 & 0.299 & 1.05 & $\mathrm{~g}$ & - & o & - & 11772 & 30 & 0.05 & 11772 & 30 & 2012 ApJS..199_..26H & - & - & - \\
\hline $00033726+6919064$ & 118.707 & 6.840 & 0.808 & 1.12 & $\mathrm{~g}$ & - & o & - & 6864 & 105 & 0.03 & 6864 & 105 & 2012ApJS..199...26H & - & - & - \\
\hline $00034142+7036434$ & 118.955 & 8.110 & 0.789 & 0.87 & $\mathrm{~g}$ & - & o & - & 4620 & 16 & 0.02 & 4620 & 16 & 2012 ApJS..199_..26H & - & - & - \\
\hline $00053428+5337528$ & 116.084 & -8.629 & 0.417 & 1.01 & $\mathrm{~g}$ & - & $\mathrm{h}$ & - & 9523 & 7 & 0.04 & - & - & - & 9523 & 7 & 1998A\&AS..130..333T \\
\hline $00083102+5301491$ & 116.417 & -9.298 & 0.411 & 1.02 & $\mathrm{~g}$ & - & o & - & 12124 & 38 & 0.05 & 12124 & 38 & 2012ApJS..199_..26H & $=$ & - & - \\
\hline $00083403+5530408$ & 116.844 & -6.853 & 0.432 & 1.05 & $\mathrm{~g}$ & - & $\mathrm{h}$ & - & 5155 & 4 & 0.02 & - & - & - & 5155 & 4 & $2003 \mathrm{~A} \& \mathrm{~A} \ldots 412 \ldots 57 \mathrm{P}$ \\
\hline $00103893+5325040$ & 116.800 & -8.968 & 0.265 & 1.00 & $\mathrm{~g}$ & - & o & - & 9900 & 23 & 0.04 & 9900 & 23 & 2012 ApJS..199_..26H & - & - & - \\
\hline $00141253+7036448$ & 119.824 & 7.966 & 1.014 & 0.92 & $\mathrm{~g}$ & - & o & * & 6958 & 8 & 0.03 & 6973 & 43 & 2012ApJS..199...26H & 6958 & 8 & 2018MNRAS.481.1262K \\
\hline $00161976+7025219$ & 119.974 & 7.753 & 1.046 & 0.43 & $\mathrm{~g}$ & $o$ & o* & - & * & * & 0.15 & * & * & 20182MZO.FLWO.0000M & - & - & - \\
\hline
\end{tabular}


18 A.C. Schröder et al.

APPENDIX B: VARIATION OF F-VALUES

ACROSS THE ZOA FOR THE PLANCK

EXTINCTION MAPS

This paper has been typeset from a $\mathrm{T}_{\mathrm{E}} \mathrm{X} / \mathrm{L} \mathrm{LT}_{\mathrm{E}} \mathrm{X}$ file prepared by the author. 


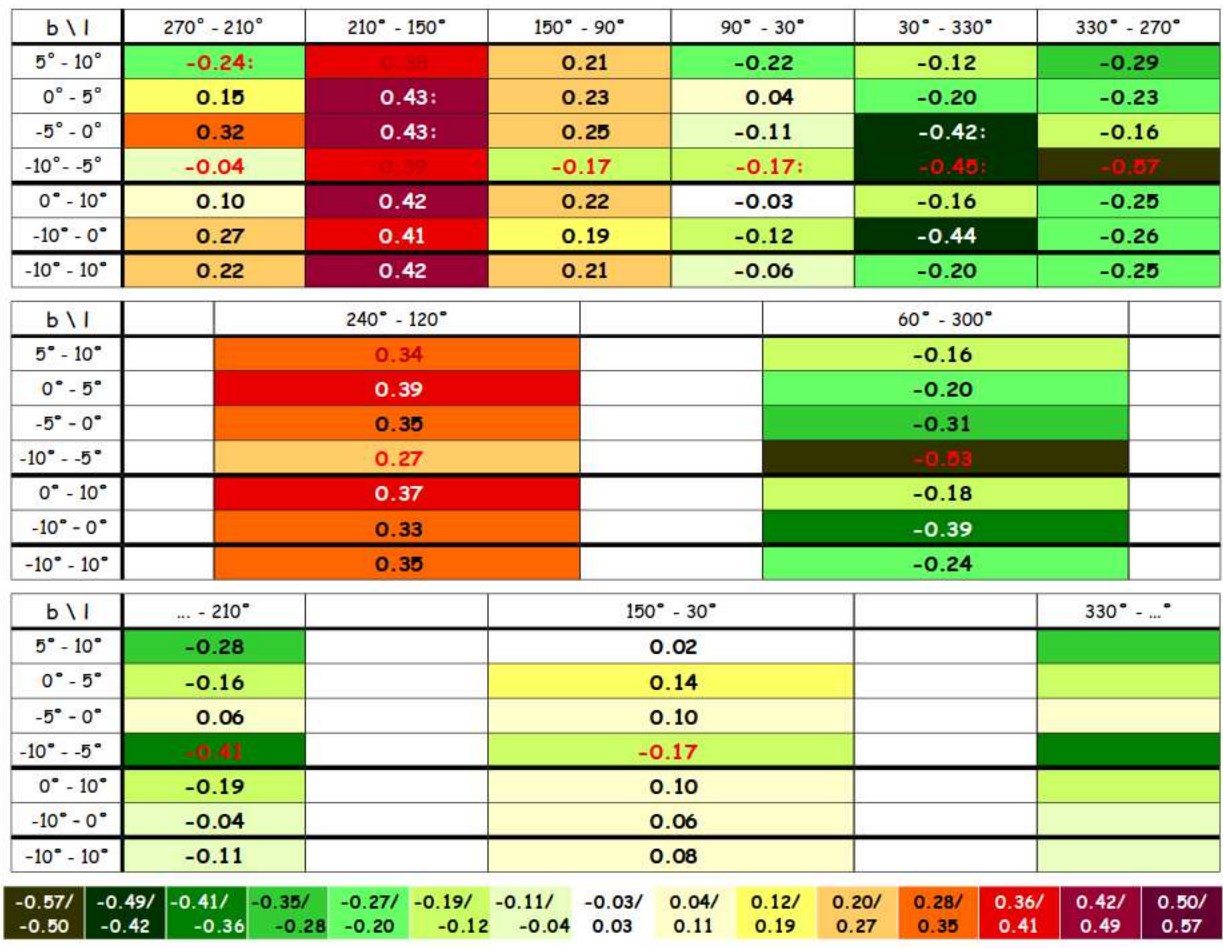

Figure B1. Same as Fig. 11 for the P-PR1 extinctions. Note the change to the colour scale.

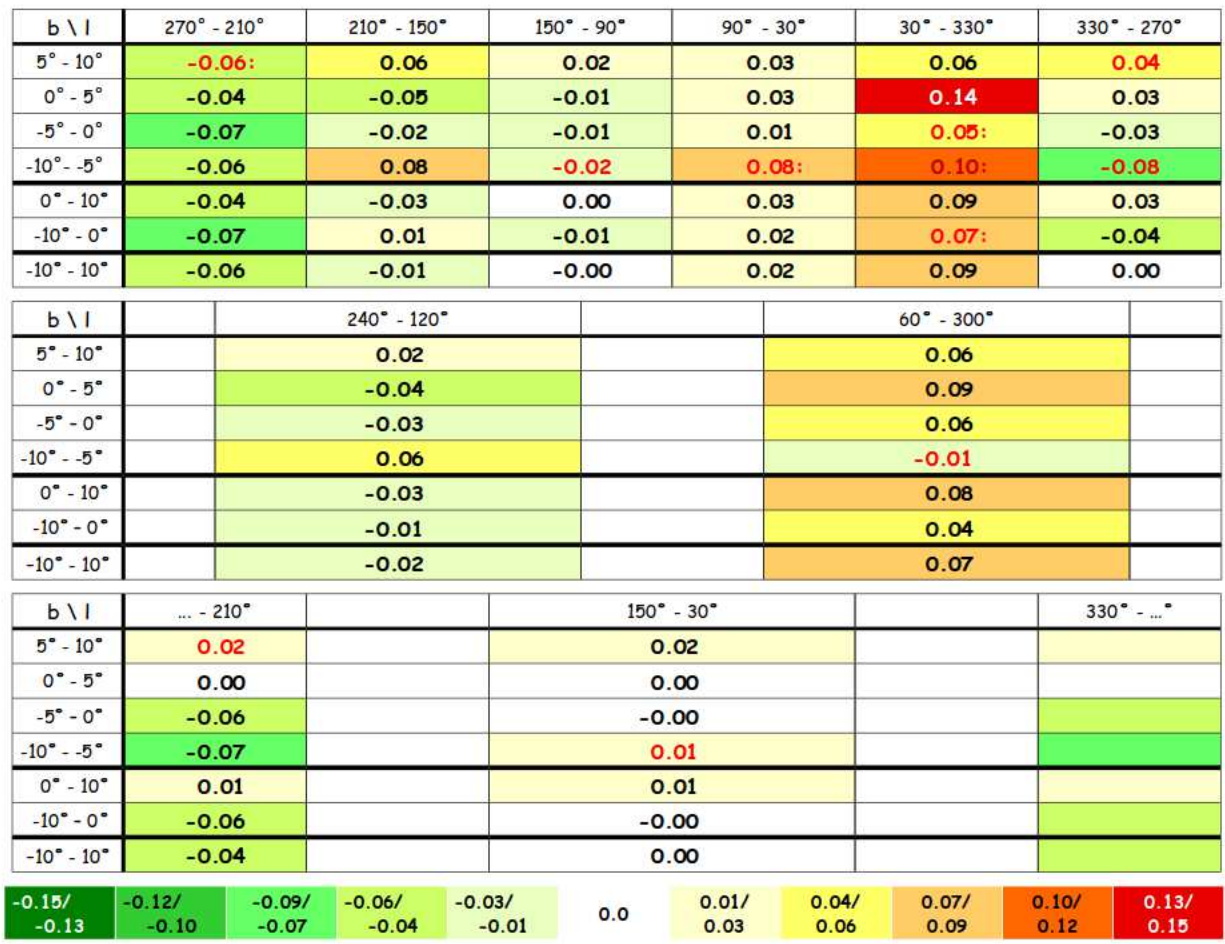

Figure B2. Same as Fig. 11 for the P-MF extinctions. 


\begin{tabular}{|c|c|c|c|c|c|c|}
\hline $\mathrm{b} \backslash \mathrm{I}$ & $270^{\circ}-210^{\circ}$ & $210^{\circ}-150^{\circ}$ & $150^{\circ}-90^{\circ}$ & $90^{\circ}-30^{\circ}$ & $30^{\circ}-330^{\circ}$ & $330^{\circ}-270^{\circ}$ \\
\hline $5^{\circ}-10^{\circ}$ & $-0.01:$ & 0.15 & 0.04 & -0.03 & -0.02 & -0.03 \\
\hline $0^{\circ}-5^{\circ}$ & 0.00 & 0.03 & 0.02 & -0.02 & -0.02 & -0.07 \\
\hline$-5^{\circ}-0^{\circ}$ & -0.01 & 0.05 & 0.02 & -0.05 & $-0.12:$ & -0.07 \\
\hline$-10^{\circ}-5^{\circ}$ & -0.02 & 0.10 & 0.01 & $0.06:$ & $-0.03:$ & \\
\hline $0^{\circ}-10^{\circ}$ & -0.00 & 0.05 & 0.03 & -0.02 & -0.02 & -0.06 \\
\hline$-10^{\circ}-0^{\circ}$ & -0.01 & 0.07 & 0.02 & -0.04 & $-0.08:$ & -0.09 \\
\hline$-10^{\circ}-10^{\circ}$ & -0.01 & 0.06 & 0.02 & -0.03 & -0.03 & -0.07 \\
\hline
\end{tabular}

\begin{tabular}{|c|c|c|c|}
\hline$b \backslash 1$ & $240^{\circ}-120^{\circ}$ & $60^{\circ}-300^{\circ}$ \\
\hline $5^{\circ}-10^{\circ}$ & 0.08 & -0.02 \\
\hline $0^{\circ}-5^{\circ}$ & 0.02 & & -0.04 \\
\hline$-5^{\circ}-0^{\circ}$ & 0.03 & -0.07 \\
\hline$-10^{\circ}--5^{\circ}$ & 0.08 & -0.11 \\
\hline $0^{\circ}-10^{\circ}$ & 0.04 & -0.03 & \\
\hline$-10^{\circ}-0^{\circ}$ & 0.04 & -0.08 & \\
\hline$-10^{\circ}-10^{\circ}$ & 0.04 & -0.04 & \\
\hline
\end{tabular}

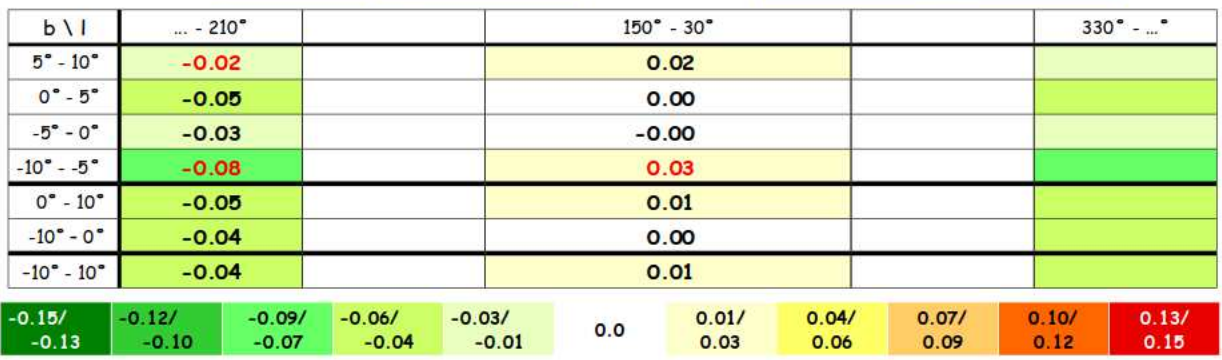

Figure B3. Same as Fig. 11 for the P-AV extinctions.

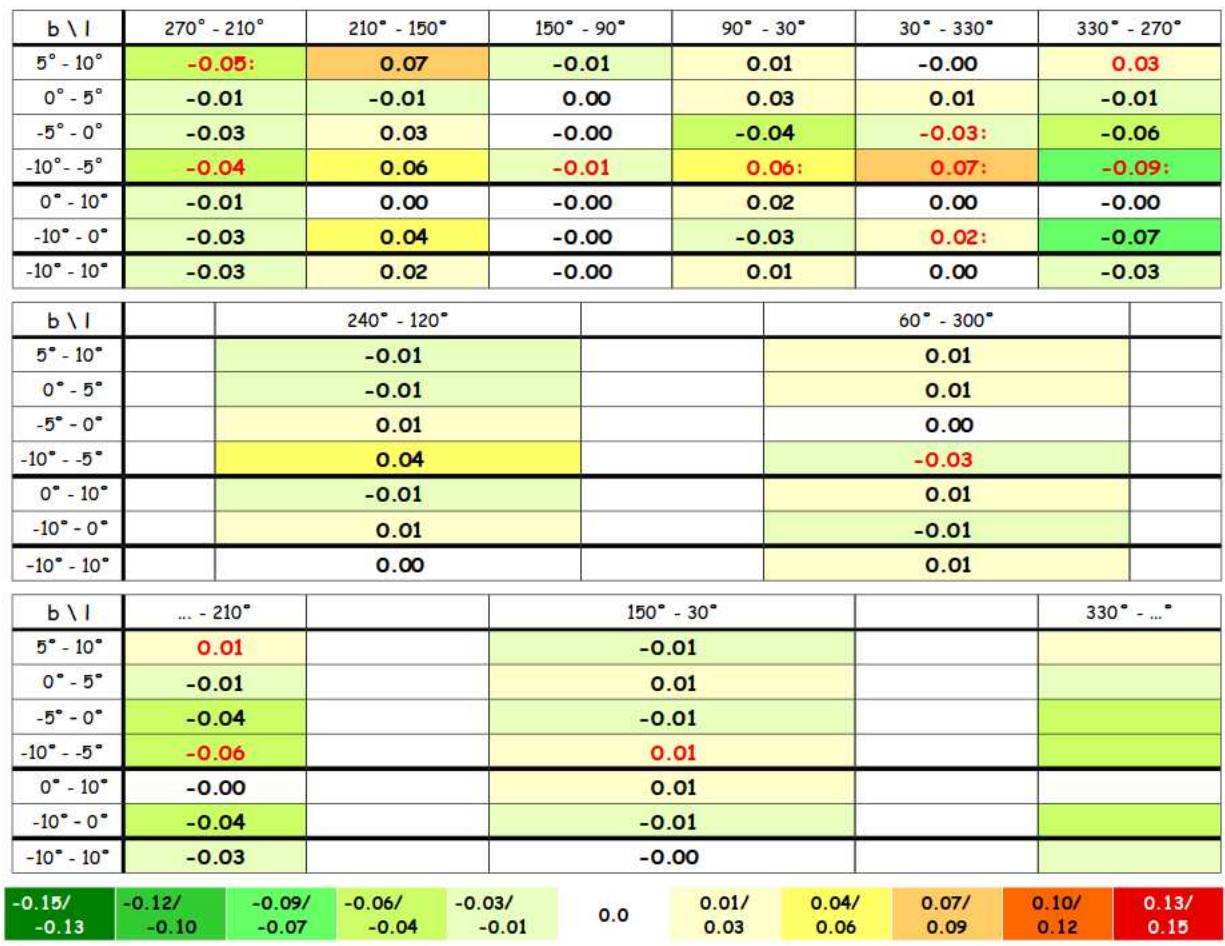

Figure B4. Same as Fig. 11 for the P-GNILC extinctions. 